\title{
COUNTING FEYNMAN-LIKE GRAPHS: QUASIMODULARITY AND SIEGEL-VEECH WEIGHT
}

\author{
ELISE GOUJARD AND MARTIN MÖLLER
}

\begin{abstract}
We prove the quasimodularity of generating functions for counting torus covers, with and without Siegel-Veech weight. Our proof is based on analyzing decompositions of flat surfaces into horizontal cylinders. The quasimodularity arise as contour integral of quasi-elliptic functions. It provides an alternative proof of the quasimodularity results of Bloch-Okounkov, Eskin-Okounkov and Chen-Möller-Zagier, and generalizes the results of BöhmBringmann-Buchholz-Markwig for simple ramification covers.
\end{abstract}

\section{Contents}

1. Introduction

2. Counting Covers of elliptic curves by global graphs

3. Shifted symmetric polynomials and completed cycles 12

4. Hurwitz numbers and graph sums 13

5. Constant coefficients of quasi-elliptic functions 17

6. Quasimodularity of graph sums 25

7. Siegel-Veech constants 29

8. Tropical covers and quasimodularity graph by graph 34

9. Examples 36

References

\section{INTRODUCTION}

The generating series counting the number of torus coverings first attracted attention with Dijkgraaf's work (Dij95]) on mirror symmetry for elliptic curves. It was shown rigorously by Kaneko-Zagier ( $[$ KZ95 $)$ that these functions are quasimodular forms. This statement was generalized by Eskin-Okounkov ([E001]) from simple branch points to arbitrary branching profile.

In this paper we show that the quasimodularity property of counting functions generalizes in two ways. We first analyze to which extent the quasimodularity holds when counting the contributions of each underlying global graph separately. The precise statement requires a correspondence theorem between covers and decorated graphs. In the case of simple branching, the global graphs are trivalent and sometimes referred to as Feynman graphs. In this case our correspondence theorem boils

Research of the first author is supported by the Fondation Mathématique Jacques Hadamard. Research of the second author is partially supported by the DFG-project MO 1884/1-1. 
down to the correspondence theorem for tropical Hurwitz numbers of torus covers proved in BBBM13, as we explain in Section 8 .

The second generalization counts coverings with a Siegel-Veech weight, motivated by Siegel-Veech constants for flat surfaces. Here again, our method provides a different approach and a refinement of the quasimodularity shown in CMZ16, by counting the contributions of each ("Feynman") graph separately.

We give some motivation for why we care about quasimodularity statements. Obviously, knowing the first few coefficients of a quasimodular form determines the whole series and thus provides a computational approach to the counting problems. Second, the asymptotic behaviour of the coefficients of a quasimodular form is wellunderstood ([CMZ16, Section 9]). Those coefficient asymptotics are important e.g. to compute the Masur-Veech volumes of moduli spaces of flat surfaces. Despite some recent advances ([AEZ16], Gou16], CMZ16]) many refined questions, concerning e.g. large genus asymptotics, spin structure distinction and Masur-Veech volumes of quadratic differential spaces in general, are still wide open. We plan to apply the techniques presented here to these cases in a sequel to this paper.

A covering $p: X \rightarrow E$ of the square torus provides $X$ with a flat metric $\omega=p^{*} \omega_{E}$. The flat surface $(X, \omega)$ is swept out by horizontal cylinders. We obtain the global graph of the covering by letting the vertices be the branch points of $p$ and the edges these horizontal cylinders. Our correspondence theorem shows roughly that decorating the graph with widths and heights at the edges and with local data (triple Hurwitz numbers) at the vertices defines a bijection with torus covers, see Proposition 2.4 for the precise statement. In the case of simple branching, our global graphs are the tropical covers of e.g. BBBM13.

For the counting problems the following special case of Theorem 5.8 is the core of the quasimodularity statements.

Theorem 1.1. Let $P$ be a product of derivatives $\wp^{(m)}\left(z_{i}-z_{j}\right)$ of the Weierstrass

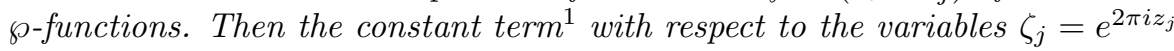

$$
\left[\zeta_{n}^{0}, \ldots, \zeta_{1}^{0}\right] P=\frac{1}{(2 \pi i)^{n}} \oint_{0+i \varepsilon_{n}}^{1+i \varepsilon_{n}} \cdots \oint_{0+i \varepsilon_{1}}^{1+i \varepsilon_{1}} P\left(z_{1}, \ldots, z_{n} ; \tau\right) d z_{1} \ldots d z_{n}
$$

is a quasimodular form. More precisely, if $P$ consists of $\ell$ factors, where the $k$-th factor involves the $m_{k}$-th derivative, then the quasimodular form has mixed weight less or equal to $\sum_{k=1}^{\ell}\left(2+m_{k}\right)$.

Note that in general these constant terms are not of pure weight 2 as we show in the example in Section [5.4, even if all $m_{k}=0$. The statement of the theorem above involves only the elliptic Weierstraß $\wp$-function. Nevertheless our proof requires Theorem 5.8 about the quasimodularity of constants terms for quasi-elliptic functions in full generality, since taking the coefficient $\left[\zeta_{1}^{0}\right]$ of an elliptic function may no longer be elliptic.

We let $N^{\circ}(\Pi)=\sum N_{d}^{\circ}(\Pi) q^{d}$ be the generating series of torus covers with branching profile $\Pi$. Our first geometric application is an independent proof of the following result of Kaneko-Zagier and Eskin-Okounkov, based on Theorem 1.1

\footnotetext{
${ }^{1}$ We refer to Section 5.3 for the conventions on the "heights" $\varepsilon_{i}$ of integration paths.

${ }^{2}$ Purity of the weight of the quasimodular form is claimed in Theorem 3.2 of [BBBM13, but it relies on Proposition 3.3, which has a gap.
} 
Theorem 1.2. (= Corollary 6.2) For any ramification profile $\Pi$ the counting function $N^{\circ}(\Pi)$ for connected torus covers of profile $\Pi$ is a quasimodular form of mixed weight less or equal to $|\Pi|+\ell(\Pi)$.

The generating series for torus covers $N^{\circ}(\Pi)=\sum_{\Gamma} N^{\circ}(\Gamma, \Pi)$ can be decomposed as according to the associated global graph $\Gamma$. In general, the individual contributions $N^{\circ}(\Gamma, \Pi)$ are not quasimodular forms. Already genus two surfaces and $\Pi$ consisting of a 3 -cycle provides an example, see Section 9.1. Our method of proof gives a refinement of the quasimodularity statement for the case of simple branch points. Along with the interpretation in terms of tropical covers we show in Section 8

Theorem 1.3 (= Corollary 8.4). In the case $\Pi=((2), \ldots,(2))$, for any trivalent graph $\Gamma$ the contribution $N^{\prime}(\Pi, \Gamma)$ of the graph $\Gamma$ to the total counting is a quasimodular form of mixed weight at most $|\Pi|+\ell(\Pi)$.

Siegel-Veech constants measure the asymptotic number of immersed cylinders in a flat surface of bounded length of the waist curve. They are important characteristic quantities of the dynamics of billiards and flat surfaces, see Section 7.1 for a brief summary and [EM01], [EMZ03, [CMZ16, Section 1] for more details. The Siegel-Veech constants for a general flat surface in a given stratum can be computed by determining the asymptotics of Siegel-Veech constants for spaces of torus covers. This in turn requires counting torus covers with a combinatorial constant, the Siegel-Veech weight (depending on an integer parameter $p \geq-1$ ), that we define in Section 7 . In analogy with the simple counting problem we consequently define the generating series $c_{p}^{\circ}(\Pi)$ of Siegel-Veech weighted coverings. Showing that a such series is a quasimodular form is important because of the good control of the coefficient asymptotics of quasimodular forms (see [CMZ16, Section 9]). Counting Siegel-Veech weighted graphs gives a new proof of the following theorem (see CMZ16, Theorem 6.4]) and the refinement graph by graph in the trivalent case that we state in Corollary 8.4

Theorem 1.4. [= Corollary 7.2 For any ramification profile $\Pi$ and any odd integer $p \geq-1$ the generating series $c_{p}^{\circ}(\Pi)$ for counting connected covers with $p$-SiegelVeech weight is a quasimodular form of mixed weight at most $|\Pi|+\ell(\Pi)+p+1$.

We conclude with an outline of the proof of Theorem 1.2 and Theorem 1.4 Using the correspondence theorem Proposition 2.4 our problem is converted into counting decorated graphs whose vertex labels are triple Hurwitz numbers. These are piecewise polynomials in the input data, i.e. the edge labels of the graph. If these were globally polynomials (as they are in the trivalent case), the graph sums can be interpreted as the constant coefficient of a polynomial in the Weierstraß $\wp-$ function and its derivatives, see Proposition 6.7. The polynomiality can be restored using completed cycles $p_{k}$ instead of the weighted symmetric group characters $f_{k}$ in the Burnside formula for counting coverings. Using the notion of $q$-bracket and the fact that both $f_{k}$ and $p_{k}$ generate the algebra of shifted symmetric functions, the arguments of Section 4.1 allow to come back to the true counting problem while maintaining quasimodularity.

Acknowledgements. We are very grateful to Alex Eskin for sharing with us the manuscript of an old project with Andrei Okounkov that was at the origin of the 
notion of completed cycles (see the reference to [12] in [OP06]). We also thank Don Zagier for many fruitful conversations on quasimodular forms and Dmitry Zvonkine and Kathrin Bringmann for useful suggestions and comments. We moreover thank the referee whose comments helped to improve the exposition of the paper.

This research was conducted at the Max-Planck-Institut für Mathematik, Bonn, whose hospitality we gratefully acknowledge.

Notation. For a partition $\lambda=\left(\lambda_{1} \geq \lambda_{2} \geq \cdot\right)$ we let $|\lambda|=\sum_{i \geq 0} \lambda_{i}$ be the number that $\lambda$ is a partition of, i.e., $\lambda \vdash|\lambda|$. We denote by $\ell(\lambda)=\max \left(\left\{i: \lambda_{i} \geq 0\right\}\right)$ the length of the partition. We also need the weight $\mathrm{wt}(\lambda)=|\lambda|+\ell(\lambda)$ of a partition. We adopt the corresponding notation for tuples, i.e. if $\mathbf{w}=\left(w_{1}, \ldots, w_{n}\right)$ then $|\mathbf{w}|=\sum w_{i}$ and $n=\ell(\mathbf{w})$.

\section{Counting Covers of elliptic curves by global Graphs}

In this section we recall basic facts about enumeration of covers, both for torus coverings and coverings of the projective line with three marked points. The aim of this section is the correspondence theorem Proposition 2.4 that gives a bijection between torus coverings and decorated graphs. This proposition holds on the level of covers without unramified components only, but (for general branching profile) neither on the level of connected coverings nor on the level of all coverings. This fact requires us to set up quite a bit of notation before giving the statement.

2.1. Covers of elliptic curves and their Hurwitz tuples. Here we recall basic facts about enumeration of covers using tuples of elements in the symmetric group. Our aim here is to explain the passage between the number of connected and nonconnected coverings and to express these numbers in terms of characters on the symmetric group. We focus on torus coverings in this section.

Let $\Pi=\left(\mu^{(1)}, \cdots, \mu^{(n)}\right)$ consist of partitions $\mu^{(i)}=\left(\mu_{1}^{(i)}, \mu_{2}^{(i)}, \cdots\right)$ such that each entry $\mu_{j}^{(i)}$ is a non-negative integer and for later use we define $g$ by $\sum_{i, j}\left(\mu_{j}^{(i)}-1\right)=$ $2 g-2$. We call such a tuple $\Pi$ a ramification profile.

A covering $p: X \rightarrow E$ of the torus $E$ has ramification profile $\Pi$, if the covering has $n$ numbered branch points and over the $i$-th branch point the sheets coming together form the partition $\mu^{(i)}$ (completed by singletons, if $\left|\mu^{(i)}\right|<\operatorname{deg}(p)$ ). Let

$$
\rho: \pi_{1}\left(E \backslash\left\{P_{1}, \ldots, P_{n}\right\}\right) \rightarrow S_{d}
$$

be the monodromy representation in the symmetric group of $d$ elements associated with a covering $p$ and some base point $P$, that we suppress in notation. We use the convention that loops (and elements of the symmetric group) are composed from right to left. The elements $\left(\alpha, \beta, \gamma_{1}, \cdots, \gamma_{n}\right)$ as in Figure1generate the fundamental group $\pi_{1}\left(E \backslash\left\{P_{1}, \ldots, P_{n}\right\}\right)$ with the relation

$$
\beta^{-1} \alpha^{-1} \beta \alpha=\gamma_{n} \cdots \gamma_{1} \text {. }
$$

Given such a homomorphism $\rho$, we let $\boldsymbol{\alpha}=\rho(\alpha), \boldsymbol{\beta}=\rho(\beta)$ and $\boldsymbol{\gamma}_{i}=\rho\left(\gamma_{i}\right)$ and call the tuple

$$
h=\left(\boldsymbol{\alpha}, \boldsymbol{\beta}, \boldsymbol{\gamma}_{1}, \cdots, \boldsymbol{\gamma}_{n}\right) \in\left(S_{d}\right)^{n+2}
$$

the Hurwitz tuple corresponding to $\rho$ and the choice of generators. Our main goal is to count coverings, or rather the corresponding Hurwitz tuples, and so we let

$$
\operatorname{Hur}_{d}(\Pi)=\left\{H=\left(\boldsymbol{\alpha}, \boldsymbol{\beta}, \boldsymbol{\gamma}_{1}, \cdots, \boldsymbol{\gamma}_{n}\right) \in\left(S_{d}\right)^{n+2} \text { of profile } \Pi\right\} \text {, }
$$



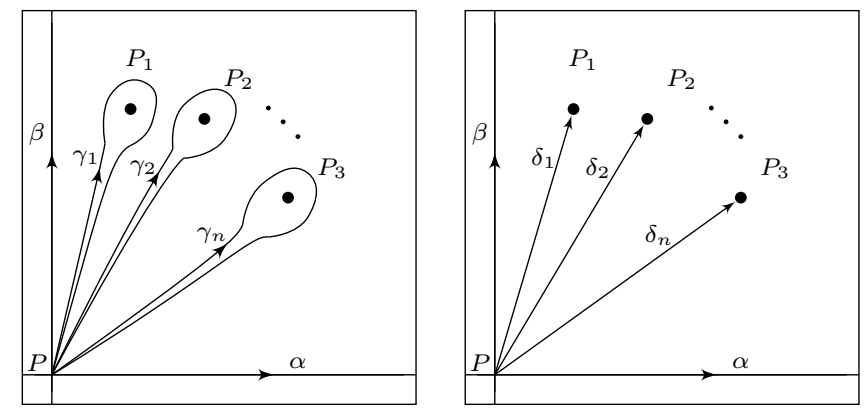

FIGURE 1. Standard presentation of $\pi_{1}\left(E \backslash\left\{P_{1}, \ldots, P_{n}\right\}\right)$

where we say that $H$ has profile $\Pi$ if the partitions $\left[\gamma_{i}\right]$ associated with conjugacy class of $\gamma_{i}$ are equal to $\mu^{(i)}$ for $i=1, \ldots, n$. Here we use the general convention to call two partitions of different sizes $d_{1} \leq d_{2}$ equal if they differ by $d_{2}-d_{1}$ parts of length one.

So far we have made no connectedness assumption, but we will ultimately be interested in counting connected coverings, hence transitive monodromy representations. We indicated this subset by an upper circle. As important technical intermediate notion we need covers without unramified components, indicated by a prime, so we let

$$
\begin{aligned}
& \operatorname{Hur}_{d}^{\circ}(\Pi)=\left\{H \in \operatorname{Hur}_{d}(\Pi):\langle H\rangle \text { acts transitively on }\{1, \ldots, d\}\right\} \\
& \operatorname{Hur}_{d}^{\prime}(\Pi)=\left\{H \in \operatorname{Hur}_{d}(\Pi):\left\langle\gamma_{1}, \cdots, \gamma_{n}\right\rangle \text { acts non-trivially on every } H \text {-orbit }\right\}
\end{aligned}
$$

The corresponding countings of covers (as usual with weight $1 / \operatorname{Aut}(p)$ ) differ from the cardinalities of these sets of Hurwitz tuples by the simultaneous conjugation of the Hurwitz tuple, hence by a factor of $d$ !. Consequently, we let

$$
N_{d}(\Pi)=\frac{\left|\operatorname{Hur}_{d}^{0}(\Pi)\right|}{d !}, \quad N_{d}^{\prime}(\Pi)=\frac{\left|\operatorname{Hur}_{d}^{\prime}(\Pi)\right|}{d !}, \quad N_{d}^{\circ}(\Pi)=\frac{\left|\operatorname{Hur}_{d}^{\circ}(\Pi)\right|}{d !},
$$

and package these data into the generating series

$$
N(\Pi)=\sum_{d=0}^{\infty} N_{d}(\Pi) q^{d}, \quad N^{\prime}(\Pi)=\sum_{d=0}^{\infty} N_{d}^{\prime}(\Pi) q^{d}, \quad N^{0}(\Pi)=\sum_{d=0}^{\infty} N_{d}^{0}(\Pi) q^{d} .
$$

From $\left|\operatorname{Hur}_{d}(\Pi)\right|=\sum_{j=0}^{d}\left(\begin{array}{l}d \\ j\end{array}\right)\left|\operatorname{Hur}_{j}^{\prime}(\Pi)\right|\left|\operatorname{Hur}_{d-j}()\right|$ one deduces that

$$
N^{\prime}(\Pi)=N(\Pi) / N() \text {. }
$$

In order to state the passage from connected counting to counting without unramified components we need to define the set of ramification points and its partitions. For $i \in\{1, \ldots, n\}$ let $\mu_{j}^{(i)}$ for $j \in J=J(i)$ be the parts of $\mu^{(i)}$ of length greater than one and let

$$
\mathcal{R}(\Pi)=\{(i, j), i \in\{1, \ldots, n\} \text { and } j \in J(i)\}
$$

be the index set of ramification points of the profile $\Pi$. We let $\mathbb{P}(\mathcal{R})$ be the set of partitions of the set $\mathcal{R}=\mathcal{R}(\Pi)$ that are finer than the partition by different first index. For any part $A$ of such a partition we let $\Pi_{A}$ be the profile consisting of the partitions $\mu_{A}^{(i)}=\left\{\mu_{j}^{(i)},(i, j) \in A\right\}$ grouped together according to $A$. We omit 
those $i$ for which there is no $j$ with $(i, j) \in A$, so that $\Pi_{A}$ is a profile with $n$ or less branch points.

Proposition 2.1. The generating function for counting covers without unramified components can be expressed in terms of counting functions for connected covers as

$$
N^{\prime}(\Pi)=\sum_{\alpha \in \mathbb{P}(\mathcal{R})} \prod_{A \in \alpha} N^{\circ}\left(\Pi_{A}\right)
$$

Proof. Any covering $p$ without unramified components induces a partition $\alpha \in \mathbb{P}(\mathcal{R})$ of the ramification points according to its connected components. We label the sheets of the covering and obtain the identity of the sets of Hurwitz numbers

$$
\left|\operatorname{Hur}_{d}^{\prime}(\Pi)\right|=\sum_{\alpha \in \mathbb{P}(\mathcal{R})} \sum_{\left(d_{A}\right)_{A \in \alpha}}\left(\begin{array}{c}
d \\
\left(d_{A}\right)_{A \in \alpha}
\end{array}\right) \prod_{A \in \alpha}\left|\operatorname{Hur}_{d_{A}}^{\circ}\left(\Pi_{A}\right)\right|,
$$

from which the claim follows immediately.

Since the summand $N^{\circ}(\Pi)$ appears on the right side, classical inclusion-exclusion allows to invert this formula and to write $N^{\circ}(\Pi)$ as a linear combination of products of $N^{\prime}\left(\Pi_{A}\right)$ for subsets $A$ of $R$.

With the aim of connecting counting problems to the representation theory of the symmetric group, we recall the classical Burnside Lemma (see e.g. LZ04, Theorem A.1.10]) that the number of Hurwitz tuples with ramification profile $\Pi$ is given by

$$
\operatorname{Hur}_{d}(\Pi)=d ! \sum_{\lambda \in \mathcal{P}(d)} \prod_{i=1}^{n} f_{\mu^{(i)}}(\lambda),
$$

where a conjugacy class $\sigma$ is completed with singletons to form a partition of $|\lambda|$ and where

$$
f_{\sigma}(\lambda)=z_{\sigma} \chi^{\lambda}(\sigma) / \operatorname{dim} \chi^{\lambda} .
$$

Here $z_{\sigma}$ denotes the size of the conjugacy class of $\sigma$ and $\operatorname{dim} \chi^{\lambda}$ is the dimension of representation $\lambda$. We also write $f_{k}$ for the special case that $\sigma$ is a $k$-cycle.

2.2. Covers of the projective line with three marked points. Covers of the projective line $\pi: S \rightarrow \mathbb{P}^{1}$ can, of course, also be described by their monodromy. The main point here is to introduce some notation and to highlight the fact that we consider one of the points $(z=1)$ to have a fixed ramification profile given by a partition $\mu$, whereas the ramification over the other two branch points $(z=0$ and $z=\infty$ ) are prescribed by two ('input' and 'output') tuples of variables $\mathbf{w}^{-}=$ $\left(w_{1}^{-}, \ldots, w_{n^{-}}^{-}\right)$and $\mathbf{w}^{+}=\left(w_{1}^{+}, \ldots, w_{n^{+}}^{+}\right)$.

We conclude again, with the passage between the number of connected and nonconnected coverings and expressions in terms of characters of the symmetric group.

The use of the terminology double and triple Hurwitz numbers is not completely consistent in the litterature. Most classically, double Hurwitz numbers count coverings with prescribed behaviour over two points and besides only simple branching. In e.g. SSZ12] (that we will use later), this notion is generalized allowing instead of simple branching several cycles of fixed length $r$. We call these generalized double Hurwitz numbers. We also need triple Hurwitz numbers that count covers with three prescribed ramification points. In the literature simple branch points might be allowed besides, but we will not need this case and do not consider it. We will frequently use the special case of triple Hurwitz numbers where the ramification 
profile over one of the points is a cycle. These are obviously (special cases of) generalized double Hurwitz numbers.

Our general notation convention is that Cov denotes a set of coverings, TR ('triple ramification') is the set of tuples describing the monodromy of a covering and $A$ denotes the number of coverings, always with weight $1 / \operatorname{Aut}(\pi)$. We use here the upper indices $\circ$ and prime as in the previous section, to denote connected covers and covers without unramified components, respectively.

We need to highlight one more detail, the numbering of preimages of the branch points. Suppose that $\operatorname{deg}(\pi)=d$. For a partition $\mu$ and a point $x \in \mathbb{P}^{1}$ we write $\pi^{-1}(x)=[\mu]$ if the cycles in $\pi^{-1}(x)$ agree with the partition $\mu$, completed by ones to form a partition of $d$. We say that $x$ has unnumbered profile $\mu$ in this case. If $\mathbf{w}$ is a tuple of integers with $\sum w_{i}=d$, we may consider it as a partition $[\mathbf{w}]$ and write $\pi^{-1}(x)=[\mathbf{w}]$ to specify an unnumbered profile. More frequently we will write that covering $\pi$ has the property $\pi^{-1}(x)=\mathbf{w}$ and we will say that $x$ has numbered profile $\mathbf{w}$ over $x$, if the covering comes with a labeling $\sigma_{x}$ of $\pi^{-1}(x)$ such that at the $i$-th point $w_{i}$ sheets come together.

We will consider most of the time the profile over $z=0$ and $z=\infty$ to be numbered and over $z=1$ to be unnumbered. If all of these points have unnumbered profiles, we add the subscript $u n$.

Consequently, we have explained the conventions for our notations

$$
\begin{aligned}
\operatorname{Cov}_{u n}\left(\mathbf{w}^{-}, \mathbf{w}^{+}, \mu\right)=\left\{\pi: S \rightarrow \mathbb{P}^{1}, \operatorname{deg}(\pi)=\sum w_{i}^{+}=\sum w_{i}^{-},\right. \\
\left.\pi^{-1}(1)=[\mu], \pi^{-1}(0)=\left[\mathbf{w}^{-}\right], \pi^{-1}(0)=\left[\mathbf{w}^{-}\right]\right\}
\end{aligned}
$$

and

$$
\begin{gathered}
\operatorname{Cov}\left(\mathbf{w}^{-}, \mathbf{w}^{+}, \mu\right)=\left\{\left(\pi: S \rightarrow \mathbb{P}^{1}, \sigma_{0}, \sigma_{\infty}\right): \operatorname{deg}(\pi)=\sum w_{i}^{+}=\sum w_{i}^{-},\right. \\
\left.\pi^{-1}(1)=[\mu], \pi^{-1}(0)=\mathbf{w}^{-}, \pi^{-1}(0)=\mathbf{w}^{-}\right\}
\end{gathered}
$$

for unnumbered and numbered covering. The same notation convention is used for

$$
\begin{aligned}
\operatorname{TR}_{u n}\left(\mathbf{w}^{-}, \mathbf{w}^{+}, \mu\right) & =\left\{T=(\boldsymbol{\alpha}, \boldsymbol{\beta}, \boldsymbol{\gamma}) \in S_{d}^{3} ; \quad[\boldsymbol{\alpha}]=\left[\mathbf{w}^{-}\right],[\boldsymbol{\beta}]=\left[\mathbf{w}^{+}\right],[\boldsymbol{\gamma}]=\mu\right\} \\
\operatorname{TR}\left(\mathbf{w}^{-}, \mathbf{w}^{+}, \mu\right) & =\left\{\left(T=(\boldsymbol{\alpha}, \boldsymbol{\beta}, \boldsymbol{\gamma}), \sigma_{0}, \sigma_{\infty}\right) ; \quad[\boldsymbol{\gamma}]=\mu,[\boldsymbol{\alpha}]=\mathbf{w}^{-},[\boldsymbol{\beta}]=\mathbf{w}^{+}\right\}
\end{aligned}
$$

for Hurwitz tuples. Moreover, we define

$$
\begin{aligned}
A_{u n}\left(\mathbf{w}^{-}, \mathbf{w}^{+}, \mu\right) & =\sum_{\pi \in \operatorname{Cov} u n\left(\mathbf{w}^{-}, \mathbf{w}^{+}, \mu\right)} \frac{1}{\operatorname{Aut}(\pi)}=\frac{1}{d !}\left|\mathrm{TR}_{u n}\left(\mathbf{w}^{-}, \mathbf{w}^{+}, \mu\right)\right| \\
A\left(\mathbf{w}^{-}, \mathbf{w}^{+}, \mu\right) & =\sum_{\pi \in \operatorname{Cov}\left(\mathbf{w}^{-}, \mathbf{w}^{+}, \mu\right)} \frac{1}{\operatorname{Aut}(\pi)}=\frac{1}{d !}\left|\operatorname{TR}\left(\mathbf{w}^{-}, \mathbf{w}^{+}, \mu\right)\right|
\end{aligned}
$$

for the weighted number of Hurwitz tuples if $d=\sum w_{i}^{+}=\sum w_{i}^{-}$, and we let $A\left(\mathbf{w}^{-}, \mathbf{w}^{+}, \mu\right)=0$ if this condition does not hold.

As a consequence of the Burnside Lemma we can again write those cardinalities in terms of characters of the symmetric group as

$$
\begin{aligned}
A_{\text {un }}\left(\mathbf{w}^{-}, \mathbf{w}^{+}, \mu\right) & =\frac{z_{\mathbf{w}^{-}} z_{\mathbf{w}^{+}}}{d !^{2}} \sum_{|\lambda|=d} \chi_{\mathbf{w}^{-}}^{\lambda} \chi_{\mathbf{w}^{+}}^{\lambda} f_{\mu}(\lambda) \\
& =\left(\prod_{i} w_{i}^{-} \prod_{i} w_{i}^{+} \prod_{j} r_{j}^{-} \prod_{j} r_{j}^{+}\right)^{-1} \sum_{|\lambda|=d} \chi_{\mathbf{w}^{-}}^{\lambda} \chi_{\mathbf{w}^{+}}^{\lambda} f_{\mu}(\lambda)
\end{aligned}
$$


if $d=\sum_{i} w_{i}^{+}=\sum_{j} w_{j}^{-}$, and zero otherwise, where $r_{j}^{ \pm}$are the multiplicities of the parts of $\mathbf{w}^{ \pm}$. Correspondingly,

$$
A\left(\mathbf{w}^{-}, \mathbf{w}^{+}, \mu\right)=\frac{1}{\prod_{i} w_{i}^{-} \prod_{i} w_{i}^{+}} \sum_{|\lambda|=d} \chi_{\mathbf{w}^{-}}^{\lambda} \chi_{\mathbf{w}^{+}}^{\lambda} f_{\mu}(\lambda)
$$

if $d=\sum_{i} w_{i}^{+}=\sum_{j} w_{j}^{-}$, and zero otherwise. This formula is the point of departure for the counting problems.

As in the case of covers of elliptic curves we conclude this section with a discussion on the passage between connected and non-connected version.

Lemma 2.2. The double Hurwitz number with no ramification point over $z=1$ is

$$
A\left(\mathbf{w}^{-}, \mathbf{w}^{+}, \emptyset\right)=\frac{1}{\prod_{i} w_{i}^{+}} \delta_{\mathbf{w}^{+}}^{\mathbf{w}^{-}} .
$$

Proof. This is a straightforward application of the second orthogonality relation for characters.

Since a general disconnected cover can be decomposed as a disjoint union of a cover without unramified components and a collection of unramified covers (i.e. of cylinders), we obtain the following lemma.

Lemma 2.3. The triple Hurwitz numbers can be written in terms of triple Hurwitz numbers without unramified components for subsets of the ramification profile as

$$
A\left(\mathbf{w}^{-}, \mathbf{w}^{+}, \mu\right)=\sum_{\substack{\mathbf{u}^{+} \subset \mathbf{w}^{+}, \mathbf{u}^{-} \subset \mathbf{w}^{-} \\|\mu| \leq\left|\mathbf{u}^{-}\right|=\left|\mathbf{u}^{+}\right|}} A^{\prime}\left(\mathbf{u}^{-}, \mathbf{u}^{+}, \mu\right) A\left(\mathbf{w}^{-} \backslash \mathbf{u}^{-}, \mathbf{w}^{+} \backslash \mathbf{u}^{+}, \emptyset\right),
$$

where we have set $A(\emptyset, \emptyset, \emptyset)=1$.

Consequently, we can apply inclusion-exclusion (or Möbius inversion) to this formula and write triple Hurwitz numbers without unramified components in terms of (non-connected) Hurwitz numbers. That is, there exists a Möbius function $M\left(\mathbf{u}^{+}, \mathbf{u}^{-}, \mathbf{w}^{+}, \mathbf{w}^{-}\right)$such that

$$
A^{\prime}\left(\mathbf{w}^{-}, \mathbf{w}^{+}, \mu\right)=\sum_{\substack{\mathbf{u}^{+} \subset \mathbf{w}^{+}, \mathbf{u}^{-} \subset \mathbf{w}^{-} \\|\mu| \leq\left|\mathbf{u}^{-}\right|=\left|\mathbf{u}^{+}\right|}} M\left(\mathbf{u}^{+}, \mathbf{u}^{-}, \mathbf{w}^{+}, \mathbf{w}^{-}\right) A\left(\mathbf{u}^{-}, \mathbf{u}^{+}, \mu\right) .
$$

2.3. Global graphs and cylinder decompositions. We now suppose moreover that the base of the covering $p$ is the square torus $E=\mathbb{C} /(\mathbb{Z}+i \mathbb{Z})$ and the $p$ is a cover without unramified components. We fix the holomorphic one-form $\omega_{E}$ on $E$ with period lattice $\Lambda=\mathbb{Z}+i \mathbb{Z}$ and provide $X$ with the flat structure $\omega=p^{*} \omega_{E}$. To such a situation we will associate a graph with decorations as follows. The horizontal foliation of $\omega$ is completely periodic. We select from each homotopy class of horizontal cylinders $c$ one representative, the core curve $\gamma_{c}$. We let $X^{0}=\backslash \cup_{c} \gamma_{c}$ be the complement of all the core curves. For merely counting covers the precise location of the branch points is irrelevant. For concreteness, we use in the sequel the branch point normalization that the $i$-th branch point has fixed coordinates $z_{i}=x_{i}+\sqrt{-1} \varepsilon_{i}$ with $0 \leq \varepsilon_{1}<\varepsilon_{2}<\cdots<\varepsilon_{n}<1$ and any $x_{i} \in[0,1)$.

The global graph $\Gamma$ associated with the flat surface $\left(X, \omega=p^{*} \omega_{E}\right)$ of ramification profile $\Pi$ is the graph $\Gamma$ with $n=|\Pi|$ vertices, labeled by $1, \ldots, n=|\Pi|$, see Figure 2 

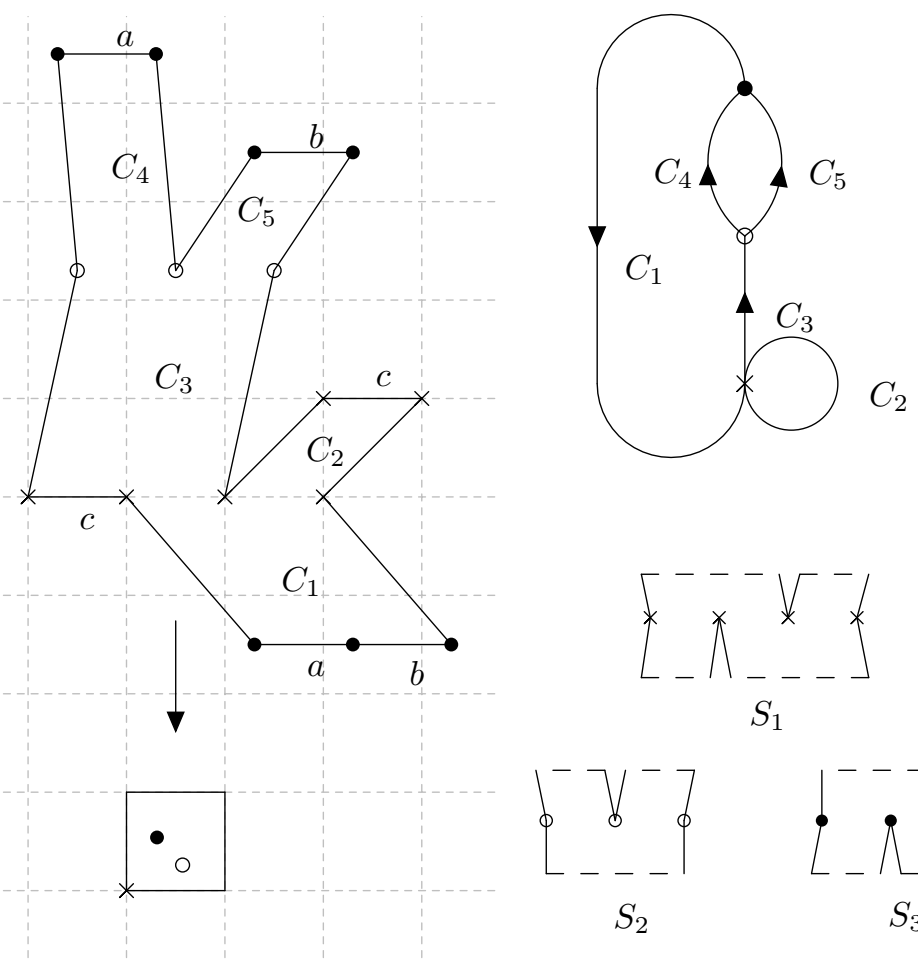

$S_{1}$

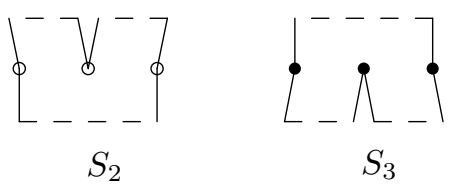

Figure 2. A torus cover, its global graph, and the local surfaces

below. The edges $E(\Gamma)$ of $\Gamma$ are in bijection with the core curves. An edge $e$ connects the vertices $i$ and $j$, if the connected components of $X^{0}$ adjacent to the core curve $\gamma(e)$ contain ramification points lying over the $i$-th and $j$-th branch point in $E$. Note that this is well-defined by the branch point normalization, which rules out that the $p$-images of two ramification points have the same height. The case $i=j$, i.e. self-edges, is of course possible.

To give an alternative definition, if each $\mu^{(i)}$ in the profile $\Pi$ is a $d$-cycle, the global graph is just the dual graph of the stable curve of the curve obtained by degenerating the surface $X$ in the horizontal direction, i.e. by applying $\operatorname{diag}\left(e^{t}, e^{-t}\right)$. In the general case, the global graph is the quotient graph of this dual graph, obtained by identifying the vertices whose corresponding branch point have the same number (in $1, \ldots, n$ ).

We provide $\Gamma$ with an orientation as follows and write $G \in \Gamma$ for the oriented graph. Fix a oriented closed loop on $E$ (e.g. a vertical straight line), intersecting the horizontal straight line once. The preimages of this loop are paths in $X$, each crossing precisely one core curve $\gamma_{c}$, and we orient the corresponding edge of $\Gamma$ in the direction of this loop. Self-edges are not given any orientation. Note that this orientation is well-defined by $p$ up to flipping all arrows of $G$.

We call the union of connected components of $X^{0}$ that carry the same label the local surfaces of $(X, \omega)$. We label these local surfaces also by an integer in $\{1, \ldots, n\}$ 
according to the ramification point they carry. This labeling is well-defined, since $p$ is a cover without unramified components.

To reconstruct a torus covering flat surface from a global graph, we need two extra data that encode the geometry of the cylinders and the geometry of the local surfaces, respectively.

Each cylinder (corresponding to an edge $e$ ) has an integral positive width $w_{e}$ and a real positive height $h_{e}$. The heights $h_{e}$ are not arbitrary, but related to the position of the branch points. For an edge $e \in E(\Gamma)$ we denote by $i^{+}(e)$ (resp. $\left.i^{-}(e)\right)$ the label of the terminal (resp. initial) vertex of the edge $e$. It is obvious from the construction that the tuple of heights $\left(h_{e}\right)_{e \in E(\Gamma)}$ belongs to the height space

$$
\widetilde{\mathbb{N}}^{E(G)}=\left\{\left(h_{e}\right)_{e \in E(\Gamma)}: h_{e}-\Delta(e) \in \mathbb{N}\right\},
$$

where $\Delta(e)=\varepsilon_{i^{+}(e)}-\varepsilon_{i^{-}(e)}$ if $i^{+}(e) \geq i^{-}(e)$ and $\Delta(e)=1+\varepsilon_{i^{+}(e)}-\varepsilon_{i^{-}(e)}$ otherwise.

The last piece of local information for a cylinder is the twist $t_{e} \in \mathbb{Z} \cap\left[0, w_{e}-1\right]$. The twist depends on the choice of a ramification point $P^{-}(e)$ and $P^{+}(e)$ in each of the two components adjacent to the cylinders and it is defined as the integer part of the real part $\left\lfloor\Re\left(\int_{s} \omega\right)\right\rfloor$ of the integral along the unique straight line joining $P^{-}(e)$ to $P^{+}(e)$ such that $t_{e} \in\left[0, w_{e}-1\right]$. The exact values of the twist will hardly matter in the sequel. It is important to retain simply that there are $w_{e}$ possibilities for the twist in a given cylinder.

We now encode the local geometry on the complement of the core curves. The restriction of the cover $p$ to any local surface is metrically a cover of an infinite cylinder, branched over one point only. Said differently, for any vertex $v \in V(\Gamma)$ the (possibly disconnected) local surface $S=S_{v}$ can be described as a covering $\pi: S \rightarrow \mathbb{P}^{1}$, ramified over $z=0, z=1$ and $z=\infty$ only. The restriction of $\omega$ to $S$ is the pullback of the infinite metric cylinder $d z / z$. The ramification profile of $\pi$ consists

- over $z=0$ of the widths $w_{e}$ of the incoming edges at $v$,

- over $z=\infty$ of the widths $w_{e}$ of the outgoing edges at $v$, and

- over $z=1$ of the subset of the branching profile $\mu^{(i)}$, where $i$ is the label of $v$.

Proposition 2.4. There is a bijective correspondence between

i) flat surfaces $(X, \omega)$ with covering $p: X \rightarrow E$ of degree $d$ of the square torus $E$ without unramified components and with $\omega=\pi^{*} \omega_{E}$, and

ii) isomorphism classes of tuples $\left(G,\left(w_{e}, h_{e}, t_{e}\right)_{e \in E(G)},\left(\pi_{v}\right)_{v \in V(G)}\right)$ consisting of

- a global graph $\Gamma$ with marked vertices and without isolated vertices together with an orientation $G \in \Gamma$,

- a collection of real numbers $\left(w_{e}, h_{e}, t_{e}\right)_{e \in E(G)}$ representing the width, height and twist of the cylinder corresponding to e. The widths $w_{e}$ are integers, the tuple of heights $\left(h_{e}\right)_{e \in E(G)} \in \widetilde{\mathbb{N}}^{E(G)}$ is in the height space, $t_{e} \in \mathbb{Z} \cap\left[0, w_{e}-1\right]$ and these numbers satisfy

$$
\sum_{e \in E(G)} w_{e} h_{e}=d
$$

- and a collection of $\mathbb{P}^{1}$-coverings $\left(\pi_{v}\right)_{v \in V(G)}$ without unramified components, with $\pi_{v} \in \operatorname{Cov}^{\prime}\left(\mathbf{w}_{v}^{-}, \mathbf{w}_{v}^{+}, \mu_{v}\right)$ where $\mathbf{w}_{v}^{-}$is the tuple of widths 
at the incoming edges at $v, \mathbf{w}_{v}^{+}$is the tuple of widths at the outgoing edges at $v$, and $\mu_{v}$ is the ramification profile given by the labels at the vertex $v$.

up to the action of the group Aut $(\Gamma)$ of automorphisms of the labeled graph $\Gamma$.

Note that an automorphism of the labeled graph $\Gamma$ preserves the vertices, i.e. it simply permutes the sets of edges sharing the same endpoints.

Proof. To each covering $p$ we can canonically associate the global graph $\Gamma$ with vertex labels according to the branch point numbering and with widths $w_{e}$ as above. The rest of the correspondence is not canonical but depends on three auxiliary choices. First, we provide $\Gamma$ with the orientation $G$ given by the upwards pointing vertical direction. Second, we move the branch points so that they satisfy the branch point normalization. Third, we choose for each edge of $\Gamma$ a pair of singularities in the local surfaces adjacent to the corresponding cylinder, one on each side.

We observe that these additional data can also obviously be recorded together with the tuples listed in ii) so that it suffices to establish a bijection with the additional data on both sides and to see that the cardinality of the forgetful maps of the additional data are the same on both sides of the correspondence.

For the second task we remark that from any point in the height space we get back by reducing mod one the heights up to a common translation (mod one) by a real number. But the heights are only well-defined up to this ambiguity of translation anyway. The number of choices of the singularities adjacent to each cylinder agrees on both sides of the correspondence, in fact this number is $\prod_{v \in V(G)} \ell\left(\mathbf{w}_{v}^{-}\right) \ell\left(\mathbf{w}_{b}^{+}\right)$.

Note that the labeling of the vertices gives a natural partial numbering of the edges, by proceeding lexicographically, i.e. first numbering all edges between the vertex one and two etc. The number of choices to complete this to a full numbering of the edges is precisely $|\operatorname{Aut}(\Gamma)|$.

The correspondence with the additional datum of an edge numbering has basically been given prior to the statement of the proposition: We use the pair of zeros adjacent to each cylinder to single out a saddle connection up to Dehn twist along the core curve of the cylinder. There is a unique representative in such a class that has holonomy $t_{e}+i h_{e}$ with $t_{e} \in \mathbb{Z} \cap\left[0, w_{e}-1\right]$. Moreover, we use the numbering of the edges to order the tuple of incoming and outgoing cylinders in each local surface, i.e. to make $\mathbf{w}^{ \pm}$an ordered tuple rather than a set of integers. This numbering also defines for every local surface an identification $\sigma_{0}$ (resp. $\sigma_{\infty}$ ) of the branch points over zero (resp. $\infty$ ) with a element in the tuple $\mathbf{w}^{-}$(resp. $\left.\mathbf{w}^{+}\right)$, so that local coverings maps $\pi_{v}$ are in $\operatorname{Cov}^{\prime}\left(\mathbf{w}_{v}^{-}, \mathbf{w}_{v}^{+}, \mu_{v}\right)$ rather than in the unordered version of this set of coverings. Note also that the prime is justified here, since the local covering is without unramified components, by the definition of components of the local surfaces as components of $X^{0}$.

For the converse correspondence, it suffices to wield together the local surfaces along a cylinder for each edge of $G$, where the identifications $\sigma_{\infty}$ of $v^{-}(e)$ and $\sigma_{0}$ of $v^{+}(e)$ determine which branches of the local surfaces are glued together. The widths $w_{e}$ and heights $h_{e}$ determine the shape of the cylinder and the twist together with the choice of a reference point on each side determines the way the cylinder is glued in. The action of an element in $\operatorname{Aut}(\Gamma)$ simply changes the $\sigma_{\infty}$ and $\sigma_{0}$ by post-composition. Consequently, any two tuples in the same Aut $(\Gamma)$-orbit give the same covering. 
Similar statements do not hold neither on the level of connected covers of graphs nor on the level of general graphs without major changes. The problem in the connected case is that some local surfaces might be disconnected while assembling to a connected flat surface in general. The problem in the disconnected case is that a covering of a local surface with an unramified component can give rise to a flat surface that is also obtained by assembling only connected local surfaces. To construct the corresponding graph without unramified components the unramified local piece has to be piled on top of the appropriate cylinder and can so be gotten rid of.

The above correspondence still gives a bijection if both sides are weighted with their automorphism group.

Proposition 2.5. In the correspondence of Proposition 2.4, an automorphism $\varphi$ of the cover $\pi$ defines a collection of automorphisms $\left(\varphi_{v}\right)_{v \in V(G)}$ of the local surfaces.

Conversely, each collection of automorphisms $\left(\varphi_{v}\right)_{v \in V(G)}$ of the local surfaces defines an automorphism $\varphi$ of the covering $\pi$.

Again, the correspondence is not canonical but depends on the choice of an auxiliary edge labeling.

Proof. Any automorphism $\varphi$ of $\pi$ preserves the marked points in $E$ and hence the vertices of $\Gamma$. Moreover, it maps cylinders to cylinders and thus induces an automorphism $\varphi_{\Gamma}$ of the graph, preserving vertices. Let $\ell: E(G) \rightarrow\{1, \ldots,|E(G)|\}$ be some auxiliary labeling of the edges of $\Gamma$. If we provide edges of the global graph of $\pi \circ \varphi$ with the labeling $\ell \circ \varphi_{\Gamma}$, then the restriction of $\varphi$ to each local surface $S_{v}$ is an automorphism $\varphi_{v}$ that preserves the labeling $\sigma_{0}$ and $\sigma_{\infty}$ of the preimages of zero and $\infty$.

The converse of this procedure obviously works as well.

\section{Shifted SymmetriC POLYNOMials AND COMPLETED CYClES}

Let $f: \mathbb{P} \rightarrow \mathbb{Q}$ be an arbitrary function on the set $\mathbb{P}$ of all partitions. Motivated by the formula (6) computing the generating function of covers without unramified components, we associate to $f$ the formal power series

$$
\langle f\rangle_{q}=\frac{\sum_{\lambda \in \mathbb{P}} f(\lambda) q^{|\lambda|}}{\sum_{\lambda \in \mathbb{P}} q^{|\lambda|}} \in \mathbb{Q}[[q]],
$$

which we will call the $q$-bracket. In the previous section, the argument was a product of functions $f_{\mu}(\cdot)$ introduced in (8) and we recall here an algebra of functions on which $q$-brackets behave nicely, as well as two generating sets for this algebrea.

The algebra of shifted symmetric polynomials is defined as $\Lambda^{*}=\lim \Lambda^{*}(n)$, where $\Lambda^{*}(n)$ is the algebra of symmetric polynomials in the $n$ variables $\lambda_{1} \leftarrow 1, \ldots, \lambda_{n}-n$. The projective limit is taken with respect to the homomorphisms setting the last variable equal to zero. One of several ways to present a partition is to list the part lengths decreasingly, i.e. a partition is given by $\lambda=\left(\lambda_{1}, \lambda_{2}, \ldots\right)$, with $\lambda_{1} \geq \lambda_{2} \geq \ldots$ and $\sum_{i=1}^{\infty} \lambda_{i}=|\lambda|$. With this notation, the functions

$$
P_{\ell}(\lambda)=\sum_{i=1}^{\infty}\left(\left(\lambda_{i}-i+\frac{1}{2}\right)^{\ell}-\left(-i+\frac{1}{2}\right)^{\ell}\right) \quad \text { and } \quad P_{\mu}=\prod_{i} P_{\mu_{i}}
$$

obviously belong to the algebra symmetric polynomials. It is also convenient to add constant terms to these function, corresponding to the regularization of the infinite 
sum, and we let

$$
p_{\ell}(\lambda)=P_{\ell}(\lambda)+\left(1-2^{-\ell}\right) \zeta(-\ell) .
$$

The name "completed cycles" refers to the functions $P_{\ell} / \ell$ for the cycles $(\ell)$ : they "complete" the functions $f_{\ell}$ defined in (8).

The following result summarizes the main properties of shifted symmetric polynomials we need. It is a combination of a theorem of Okounkov and Olshanski ([097]) and a theorem of Kerov and Olshanski.

Theorem 3.1 ([KO94]). The algebra $\Lambda^{*}$ is freely generated by all the $p_{\ell}$ (or equivalently, by the $P_{\ell}$ ) with $\ell \geq 1$. The functions $f_{\mu}$ defined in (8) belong to $\Lambda^{*}$. More precisely, as $\mu$ ranges over all partitions, these functions $f_{\mu}$ form a basis of $\Lambda^{*}$.

To convert from the $P_{\ell}$ to the $f_{\mu}$, note that $f_{1}=P_{1}$ and $f_{2}=P_{2} / 2$, and more generally $f_{\ell}$ starts with $P_{\ell} / \ell$, for example

$$
\begin{aligned}
& f_{3}=\frac{1}{3} P_{3}-\frac{1}{2} P_{1}^{2}+\frac{5}{12} P_{1}, \quad f_{4}=\frac{1}{4} P_{4}-P_{1} P_{2}+\frac{11}{8} P_{2} \\
& f_{5}=\frac{1}{5} P_{5}-P_{3} P_{1}-\frac{1}{2} P_{2}^{2}+\frac{5}{6} P_{1}^{3}-\frac{15}{4} P_{1}^{2}+\frac{19}{6} P_{3}+\frac{189}{80} P_{1} .
\end{aligned}
$$

We refer to [PP06, Section 3.3] or [Las08 for the conversion formulas in general.

At this stage we mention the following important result of Bloch-Okounkov and refer to Section 5.1 for the definition of quasimodular forms. Using Theorem 3.1 one provides the algebra $\Lambda^{*}$ with a weight grading by assigning $p_{\ell}$ the weight $k=\ell+1$.

Theorem 3.2 ([BO0] $)$. If $f$ is a shifted symmetric function of weight $k$, then $\langle f\rangle_{q}$ is a quasimodular form of weight $k$.

There exists a long list of quite different proofs of this theorem. Already BO00 contains two proofs, one in the spirit of KZ95 and one with an explicit formula using determinants of theta derivatives. A proof based on vertex operators is given in Mil03. Zagier ( Zag16) gave a very short proof, that also provided an efficient recursive method to compute $q$-brackets.

In this paper we will not use the Bloch-Okounkov theorem, but rather give yet another proof, by counting graphs with weights, at the end of Section 6 . This proof is not our main objective and the proof is rather roundabout, but it shows that a lot of quasimodularity results can be ultimately traced back to Theorem 5.8 .

\section{HuRWitz NUMBERS AND GRAPH SUMS}

The first part of the section is purely expository and we recall some known (piece-wise) polynomiality properties of the cardinalities of the Hurwitz numbers introduced in (12) and its completed cycle variants. Then we combine the definition of triple Hurwitz numbers with the composition of torus covers into global graphs and local surfaces to obtain a formula for counting torus covers in terms of a graph count of triple Hurwitz numbers.

4.1. Triple Hurwitz numbers with completed cycles. In general, the generalized double Hurwitz numbers with completed cycles are only piecewise polynomials in variables $w_{i}^{-}$and $w_{i}^{+}$on the chambers defined by the walls where a partial sum of the $w_{i}^{-}$agrees with a partial sum of the $w_{i}^{+}$. Recall that the shifted symmetric function $f_{\ell}$ satisfies $f_{\ell}=\frac{1}{\ell} P_{\ell}+\cdots$. The formal triple Hurwitz numbers with 
completed cycles

$$
\bar{A}\left(\mathbf{w}^{-}, \mathbf{w}^{+}, \mu\right)=\frac{1}{\prod_{i} w_{i}^{-} \prod_{i} w_{i}^{+}} \sum_{|\lambda|=d} \chi_{\mathbf{w}^{-}}^{\lambda} \chi_{\mathbf{w}^{+}}^{\lambda} \frac{P_{\mu}(\lambda)}{\prod \mu_{i}}
$$

obtained by replacing $f_{\mu}$ by the completed cycles $P_{\mu} / \prod \mu_{i}$ has much better properties, e.g. it is a polynomial outside the walls if $\mu=\left(\mu_{1}\right)$ is a partition consisting of a single cycle. To remove the jumps on the walls we introduce the triple Hurwitz numbers with completed cycles and without unramified components

$$
\bar{A}^{\prime}\left(\mathbf{w}^{-}, \mathbf{w}^{+}, \mu\right)=\sum_{\substack{\mathbf{u}^{+} \subset \mathbf{w}^{+}, \mathbf{u}^{-} \subset \mathbf{w}^{-} \\|\mu| \leq\left|\mathbf{u}^{-}\right|=\left|\mathbf{u}^{+}\right|}} M\left(\mathbf{u}^{+}, \mathbf{u}^{-}, \mathbf{w}^{+}, \mathbf{w}^{-}\right) \bar{A}\left(\mathbf{u}^{-}, \mathbf{u}^{+}, \mu\right) .
$$

by applying the same inclusion-exclusion inversion to $\bar{A}$ as we $\operatorname{did}$ in (17) to $A$. The main reason to introduce completed cycles here is the following polynomiality result when $\mu$ is a cycle and triple Hurwitz numbers are in fact generalized double Hurwitz numbers. We learned about this through draft notes of Okounkov. It can be combined from results of Shadrin, Spitz and Zvonkine in [SSZ12].

Theorem 4.1. If $\mu_{1}+1-\ell\left(\mathbf{w}^{-}\right)-\ell\left(\mathbf{w}^{+}\right)$is even, then the triple Hurwitz numbers $\bar{A}^{\prime}\left(\mathbf{w}^{-}, \mathbf{w}^{+},\left(\mu_{1}\right)\right)$ with completed cycles and without unramified components for the last argument $\mu=\left(\mu_{1}\right)$ being a partition consisting of a single part is an even polynomial in the variables $w_{i}^{-}$and $w_{i}^{+}$.

$$
\text { If } \mu_{1}+1-\ell\left(\mathbf{w}^{-}\right)-\ell\left(\mathbf{w}^{+}\right) \text {is odd, then } \bar{A}^{\prime}\left(\mathbf{w}^{-}, \mathbf{w}^{+},\left(\mu_{1}\right)\right)=0 \text {. }
$$

Proof. The completed generalized double Hurwitz numbers are piecewise polynomial functions for any number of ramification points besides the two prescribed ones, but the polynomiality is global for one ramification point, as we now explain in detail.

Fix $m=\ell\left(\mathbf{w}^{-}\right)$and $n=\ell\left(\mathbf{w}^{+}\right)$. We consider the vector space $V=\left\{\left(\mathbf{w}^{-}, \mathbf{w}^{+}\right)\right.$: $\left.\left|\mathbf{w}^{-}\right|=\left|\mathbf{w}^{+}\right|\right\}$and for $I \subset\{1, \ldots, m\}$ and $J \subset\{1, \ldots, n\}$ we define the hyperplane

$$
W_{I, J}=\left\{\left(\mathbf{w}^{-}, \mathbf{w}^{+}\right) \in V ;\left|\mathbf{w}_{I}^{-}\right|-\left|\mathbf{w}_{J}^{+}\right|=0\right\} .
$$

The sets $W_{I, J}$ are the walls of a hyperplane arrangement. In the interior of the chambers, the connected and disconnected Hurwitz numbers obviously coincide. For a chamber $\mathfrak{c}$ of this arrangement, Theorem 6.4 of [SSZ12 shows that $\bar{A}\left(\mathbf{w}^{-}, \mathbf{w}^{+},\left(\mu_{1}\right)\right)_{\mid \mathfrak{c}}$ is a homogeneous polynomial of degree $\mu_{1}+1-\ell\left(\mathbf{w}^{-}\right)-\ell\left(\mathbf{w}^{+}\right)$.

The wall crossing formula (Theorem 6.6 of [SSZ12]) of two adjacent chambers $\mathfrak{c}_{1}$ and $\mathfrak{c}_{2}$ of the wall $W_{I, J}$ can be written in the case of the last argument $\mu=\left(\mu_{1}\right)$ being a partition consisting of a single part as

$$
\begin{aligned}
& \bar{A}^{\prime}\left(\mathbf{w}^{-}, \mathbf{w}^{+},\left(\mu_{1}\right)\right)_{\mid \mathfrak{c}_{1}}-\bar{A}^{\prime}\left(\mathbf{w}^{-}, \mathbf{w}^{+},\left(\mu_{1}\right)\right)_{\mid \mathfrak{c}_{2}} \\
= & \delta^{2}\left(\bar{A}^{\prime}\left(\mathbf{w}_{I}^{-}, \mathbf{w}_{J}^{+}+\delta,\left(\mu_{1}\right)\right) \bar{A}^{\prime}\left(\mathbf{w}_{I^{c}}^{-}+\delta, \mathbf{w}_{J^{c}}^{+}, \emptyset\right)\right. \\
& \left.\quad+\bar{A}^{\prime}\left(\mathbf{w}_{I^{c}}^{-}+\delta, \mathbf{w}_{J^{c}}^{+},\left(\mu_{1}\right)\right) \bar{A}^{\prime}\left(\mathbf{w}_{I}^{-}, \mathbf{w}_{J}^{+}+\delta, \emptyset\right)\right),
\end{aligned}
$$

where $\delta=\left|\mathbf{w}^{-}\right|-\left|\mathbf{w}^{+}\right|$. In fact, SSZ12 state this formula in term of $\bar{A}$ instead of $\bar{A}^{\prime}$, but for $\delta=0$ the formula holds trivially by (25), and outside the walls the covers have no unramified component. In this expression the terms with no ramification vanish since $\bar{A}^{\prime}$ denotes covers without unramified components. This 
implies that the polynomials are the same in any two adjacent chambers and hence the expression is globally polynomial.

Moreover, Theorem 6.4 of [SSZ12] implies that the polynomial $\bar{A}^{\prime}\left(\mathbf{w}^{-}, \mathbf{w}^{+},\left(\mu_{1}\right)\right)$ has the same parity as $\mu_{1}+1-\ell\left(\mathbf{w}^{-}\right)-\ell\left(\mathbf{w}^{+}\right)$. It remains to show that the polynomial vanishes when this expression is odd. The triple Hurwitz number without completed cycles $A^{\prime}\left(\mathbf{w}^{-}, \mathbf{w}^{+},\left(\mu_{1}\right)\right)$ vanishes for $\mu_{1}+1-\ell\left(\mathbf{w}^{-}\right)-\ell\left(\mathbf{w}^{+}\right)$odd by the Riemann-Hurwitz formula and the same statement holds for $f_{\mu_{1}}$ replaced by any $f_{\mu}$ with $\operatorname{wt}(\mu)$ odd. Since any $p_{\mu}$ of odd (resp. even) weight is a linear combination of $f_{\mu}$ of odd (resp. even) weight (see OP06, Formula (0.22) for the general statement) the claim follows.

Later we will need to allow more general functions on partitions, and hence we define for any function $F$ on partitions

$$
A\left(\mathbf{w}^{-}, \mathbf{w}^{+}, F\right)=\frac{1}{\prod_{i} w_{i}^{-} \prod_{i} w_{i}^{+}} \sum_{|\lambda|=d} \chi_{\mathbf{w}^{-}}^{\lambda} \chi_{\mathbf{w}^{+}}^{\lambda} F(\lambda)
$$

and we define $A^{\prime}\left(\mathbf{w}^{-}, \mathbf{w}^{+}, F\right)$ in terms of $A\left(\mathbf{w}^{-}, \mathbf{w}^{+}, F\right)$ as in (25)). In this notation we retrieve the previous definition of triple Hurwitz numbers as

$$
A^{\prime}\left(\mathbf{w}^{-}, \mathbf{w}^{+}, \mu\right)=A^{\prime}\left(\mathbf{w}^{-}, \mathbf{w}^{+}, f_{\mu}\right) \quad \text { and } \quad \bar{A}^{\prime}\left(\mathbf{w}^{-}, \mathbf{w}^{+}, \mu\right)=A^{\prime}\left(\mathbf{w}^{-}, \mathbf{w}^{+}, \frac{P_{\mu}}{\prod \mu_{i}}\right) .
$$

4.2. Graph sums with triple Hurwitz numbers. The first goal here is to use Proposition 2.4 and Proposition 2.5 to write the generating series $N^{\prime}(\Pi)$ for counting torus covers without unramified components in terms of graph sums involving triple Hurwitz numbers without unramified components. First of all, we can decompose $N^{\prime}(\Pi)$ according to the contribution of the individual graphs, i.e.

$$
N^{\prime}(\Pi)=\frac{1}{|\operatorname{Aut}(\Gamma)|} \sum_{\Gamma} N^{\prime}(\Gamma, \Pi),
$$

where the sum is over all (not necessarily connected) labeled graphs $\Gamma$ with $n=|\Pi|$ vertices and where $\operatorname{Aut}(\Gamma)$ are the automorphisms of the graph $\Gamma$ that respect the vertex labeling. (Note that $\Gamma$ has neither a labeling nor an orientation on the edges.)

Proposition 4.2. The contributions of individual labeled graphs to $N^{\prime}(\Pi)$ can be expressed in terms of triple Hurwitz numbers as

$$
N^{\prime}(\Gamma, \Pi)=\sum_{G \in \Gamma} N^{\prime}(G, \Pi)
$$

where

$$
N^{\prime}(G, \Pi)=\sum_{\substack{h \in \tilde{\mathbb{N}}^{E}(G) \\ w \in \mathbb{Z}_{+}^{E(G)}}} \prod_{e \in E(G)} w_{e} q^{h_{e} w_{e}} \prod_{v \in V(G)} A^{\prime}\left(\mathbf{w}_{v}^{-}, \mathbf{w}_{v}^{+}, \mu_{v}\right) \delta(v)
$$

and where

$$
\delta(v)=\delta\left(\sum_{i \in e_{+}(v)} w_{i}^{+}-\sum_{i \in e_{-}(v)} w_{i}^{-}\right) .
$$

The delta-function factor is redundant in this expression by our definition of $A^{\prime}\left(\mathbf{w}_{v}^{-}, \mathbf{w}_{v}^{+}, \mu_{v}\right)$, but keeping it will be important once we pass from $A^{\prime}$ to a polynomial expression. 
Proof. This is a direct consequence of the correspondence in Proposition 2.4. The degree of the covering is encoded in the $\left(w_{e}, h_{e}\right)$ by (19) and the factor $w_{e}$ accounts for the number of possible twists (values of $t_{e}$ ) for any given edge $e$.

The strategy to prove quasimodularity is to reduce to "expressions as in (27) and (28)" but with $\bar{A}^{\prime}$ as argument, which is polynomial by Theorem 4.1. To formalize this, recall from the combination of (6) and (20) that the counting function $N^{\prime}(\Pi)$ is a $q$-bracket of a shifted symmetric function. By Theorem 3.1 it hence suffices to treat $q$-brackets of products of the $p_{\ell}$.

Theorem 4.3. The q-bracket of any shifted symmetric function can be expressed as a graph sum

$$
\left\langle p_{\ell_{1}} \cdots p_{\ell_{n}}\right\rangle_{q}=\sum_{\Gamma} \sum_{G \in \Gamma}\left\langle p_{\ell_{1}} \cdots p_{\ell_{n}}\right\rangle_{q, G}
$$

where the sum runs over graphs with $n$ labeled vertices and all orientations $G$ of $\Gamma$, and where

$$
\left\langle p_{\ell_{1}} \cdots p_{\ell_{n}}\right\rangle_{q, G}=\sum_{\substack{h \in \tilde{\mathbb{N}}^{E(G)} \\ w \in \mathbb{Z}_{+}^{E(G)}}} \prod_{e \in E(G)} w_{e} q^{h_{e} w_{e}} \prod_{v \in V(G)} \bar{A}^{\prime}\left(\mathbf{w}_{v}^{-}, \mathbf{w}_{v}^{+},\left(\ell_{\# v}\right)\right) \delta(v) .
$$

Here \#v denotes the label of the vertex $v$.

Proof. Our strategy is to reduce this to the cases covered by Proposition 4.2 For this purpose, we define for any function $F$ on partitions the auxiliary brackets

$$
\left[F_{1}, \ldots, F_{n}\right]=\sum_{\Gamma}\left[F_{1}, \ldots, F_{n}\right]_{\Gamma}, \quad\left[F_{1}, \ldots, F_{n}\right]_{\Gamma}=\sum_{G \in \Gamma}\left[F_{1}, \ldots, F_{n}\right]_{G}
$$

where the sum is over all labeled graphs $\Gamma$ with $n$ vertices and over all the orientations, respectively, and where

$$
\left[F_{1}, \ldots, F_{n}\right]_{G}=\sum_{\substack{h \in \mathbb{N}^{E(G)} \\ w \in \mathbb{Z}_{+}^{E(G)}}} \prod_{i \in E(G)} w_{i} q^{h_{i} w_{i}} \prod_{v \in V(G)} A^{\prime}\left(w_{v}^{-}, w_{v}^{+}, F_{\# v}\right) \delta(v) .
$$

In this notation, we want to show that

$$
\left\langle p_{\ell_{1}} \cdots p_{\ell_{n}}\right\rangle_{q}=\left[p_{\ell_{1}}, \ldots, p_{\ell_{n}}\right] .
$$

On the other hand, Proposition (4.2) can be restated in this notation as

$$
\left\langle f_{\mu_{1}} \cdots f_{\mu_{n}}\right\rangle_{q}=\left[f_{\mu_{1}}, \ldots, f_{\mu_{n}}\right] .
$$

We can express by Theorem 3.1 each of these generators $p_{\ell}$ of $\Lambda^{*}$ as $p_{\ell}=\sum_{\mu} c_{\ell, \mu} f_{\mu}$ for some coefficients $c_{\ell, \mu}$. Obviously, a $q$-bracket of a product $n$ shifted symmetric functions is multilinear in the $n$ arguments. On the other hand, the brackets introduced in (32) are multilinear as well, since the arguments of the bracket appear only linearly as arguments of $A^{\prime}$. Consequently, equation (34) is a linear combination of equations of the form (35). 


\section{Constant COEFficients of QUASI-Elliptic FUnCtions}

5.1. Quasimodular forms. Kaneko and Zagier introduced the quasimodular forms in [KZ95] in connection with counting simply branched covers of the torus.

A quasimodular form for the cofinite Fuchsian group $\Gamma \subset \mathrm{SL}(2, \mathbb{R})$ of weight $k$ is a function $f: \mathbb{H} \rightarrow \mathbb{C}$ that is holomorphic on $\mathbb{H}$ and the cusps of $\Gamma$ and such that there exists and integer $p$ and holomorphic functions $f_{i}: \mathbb{H} \rightarrow \mathbb{C}$ such that

$$
(c \tau+d)^{-k} f\left(\frac{a \tau+b}{c \tau+d}\right)=\sum_{i=0}^{p} f_{i}(\tau)\left(\frac{c}{c \tau+d}\right)^{i}
$$

for all $\left(\begin{array}{ll}a & b \\ c & d\end{array}\right) \in \Gamma$.

Note that this definition implies (using the identity matrix) that $f_{0}=f$. The smallest integer $p$ with the above property is called the depth of the quasimodular forms. By definition, quasimodular forms of depth zero are simply modular forms. The basic examples of quasimodular forms are the Eisenstein series defined by

$$
G_{2 k}(\tau)=\frac{(2 k-1) !}{2(2 \pi i)^{2 k}} \sum_{(m, n) \in \mathbb{Z}^{2} \backslash\{(0,0\}} \frac{1}{(m+n \tau)^{2 k}}=-\frac{B_{2 k}}{4 k}+\sum_{n=1}^{\infty} \sigma_{2 k-1}(n) q^{n} .
$$

Here $B_{l}$ is the Bernoulli number, $\sigma_{l}$ is the divisor sum function and $q=e^{2 \pi i \tau}$. For $k \geq 2$ these are modular forms, while for $k=2$ the Eisenstein series

$$
G_{2}(\tau)=-\frac{1}{24}+\sum_{n=1}^{\infty} \sigma_{1}(n) q^{n}
$$

is a quasimodular form of weight 2 and depth 1 for $\operatorname{SL}(2, \mathbb{Z})$. Note that the $q$ expansion makes sense as a definition for $G_{2 k+1}$ but does not give a quasimodular form. We will encounter this power series in Section 9

In terms of Eisenstein series, we recall a characterization of quasimodular forms, that might serve alternatively a definition of that ring for the special case of the modular group.

Proposition 5.1 ([KZ95]). The ring of quasimodular forms for $\Gamma=\operatorname{SL}(2, \mathbb{Z})$ is equal to $\mathbb{C}\left[E_{2}, E_{4}, E_{6}\right]$, the polynomial ring over $\mathbb{C}$ generated by the first three Eisenstein series. This ring is stable under the $q$-derivative $D_{q}=q \frac{\partial}{\partial q}=\frac{1}{2 \pi i} \frac{\partial}{\partial \tau}$. More precisely, the q-derivative of a quasimodular form of weight $k$ is a quasimodular form of weight $k+2$.

5.2. Coefficients of a two-variable Jacobi form. The main player of this section is a function $F_{\tau}(u, v)$ in two "Jacobi" variables $u, v$, that was used by Zagier (Zag91]) in connection with periods of modular forms. For its definition we use the genus 1 Jacobi theta function

$$
\theta(u)=\theta(u ; \tau)=\sum_{n \in \mathbb{Z}}(-1)^{n} q^{\frac{1}{2}\left(n+\frac{1}{2}\right)^{2}} e^{\left(n+\frac{1}{2}\right) u} .
$$

We then let

$$
F_{\tau}(u, v)=\frac{\theta(u+v) \theta^{\prime}(0)}{\theta(u) \theta(v)}
$$

where we denote by prime the $u$-derivative $f^{\prime}(u)=\frac{\partial f}{\partial u}$. The main feature of $f$ is that we know both its Fourier expansion, which we will connect to the counting functions we are interested in, and the Laurent expansion, which is the main tool to prove quasimodularity statements. 
Theorem 5.2. (Zag91, Theorem 3.1]) The Fourier development of $F_{\tau}(u, v)$ is

$$
F_{\tau}(u, v)=\frac{1}{2}\left(\operatorname{coth} \frac{u}{2}+\operatorname{coth} \frac{v}{2}\right)-2 \sum_{n=1}^{\infty}\left(\sum_{d \mid n} \sinh \left(d u+\frac{n}{d} v\right)\right) q^{n} .
$$

Its Laurent series expansion is

$$
F_{\tau}(u, v)=\frac{1}{u}+\frac{1}{v}-2 \sum_{r, s=0}^{\infty} D_{q}^{\min (r, s)} G_{|r-s|+1}(\tau) \frac{u^{r}}{r !} \frac{v^{s}}{s !} .
$$

The function $F_{\tau}(u, v)$ has the elliptic transformation property

$$
F_{\tau}(u+2 \pi i(n \tau+s), v+2 \pi i(m \tau+r))=q^{-m n} \zeta^{-m} \eta^{-n} F_{\tau}(u, v),
$$

for all $m, n, r, s \in \mathbb{Z}$, where $\zeta=e^{u}$ and $\eta=e^{v}$, and the modular transformation property

$$
F_{\frac{a \tau+b}{c \tau+d}}\left(\frac{u}{c \tau+d}, \frac{v}{c \tau+d}\right)=(c \tau+d) e^{\frac{c u v / 2 \pi i}{c \tau+d}} F_{\tau}(u, v)
$$

for all $\left(\begin{array}{ll}a & b \\ c & d\end{array}\right) \in \mathrm{SL}(2, \mathbb{Z})$.

In the sequel, we will use three functions derived from coefficients of $F_{\tau}(u, v)$, namely

$$
Z(z)=-\left[v^{0}\right] F_{\tau}(u, v), \quad P(z)=Z^{\prime}(z), \quad L(z)=-\left[v^{1}\right] F_{\tau}(u, v)+\frac{1}{12}
$$

where $u=2 \pi i z$. The first one, $Z(z)=-\frac{\theta^{\prime}(2 \pi i z)}{2 \pi i \theta(2 \pi i z)}=-\zeta(z) / 2 \pi i+2 G_{2} 2 \pi i z$ is the classical Weierstraß $\zeta$-function up to normalization and an additive term, the second is $P(z)=\frac{1}{(2 \pi i)^{2}} \wp(z)+2 G_{2}$ is the Weierstra $\wp \wp$-function up to normalization and an additive term. The last one has no classical name but it is the function that makes the extension to Siegel-Veech weighted counting work. A direct consequence of Theorem 5.2 are the Fourier developments in the domain $|q|<|\zeta|<1$

$$
\begin{aligned}
Z(z) & =\frac{1}{2}+\sum_{k \geq 1}\left(\zeta^{k}+\sum_{n \geq 1} q^{n k}\left(\zeta^{k}-\zeta^{-k}\right)\right) \\
P(z) & =\sum_{k \geq 1}\left(k \zeta^{k}+\sum_{n \geq 1} k q^{n k}\left(\zeta^{k}+\zeta^{-k}\right)\right) \\
L(z) & =\sum_{k \geq 1, n \geq 1} n q^{n k}\left(\zeta^{k}+\zeta^{-k}\right)
\end{aligned}
$$

and the Laurent series developments

$$
\begin{aligned}
& Z(z)=-\frac{1}{u}+2 \sum_{k=0}^{\infty} \frac{G_{2 k+2}}{(2 k+1) !} u^{2 k+1} \\
& P(z)=\frac{1}{u^{2}}+2 \sum_{k=0}^{\infty} \frac{G_{2 k+2}}{(2 k) !} u^{2 k} \\
& L(z)=2 G_{2}+\frac{1}{12}+2 \sum_{k=1}^{\infty} \frac{D_{q} G_{2 k}}{(2 k) !} u^{2 k}
\end{aligned}
$$

of our special functions.

We refer to the shift $P$ of the Weierstrass- $\wp-$ function as the propagator. (This terminology is used in e.g. Dij95, BBBM13. It goes back to BCOV94 and the function $P$ is hence also called BCOV-propagator in e.g. Li12.) 
5.3. Quasimodular forms as constant coefficients of quasi-elliptic functions. We proceed with our main criterion for quasimodularity, involving the constant coefficients of products of the functions $Z, P$ and $L$ introduced above, and its derivatives. We start with a general remark on the domains where the expansions are valid. Suppose that the meromorphic function $f\left(z_{1}, z_{2}, \ldots, z_{n} ; \tau\right)$ is periodic under $z_{j} \mapsto z_{j}+1$ for each $j$ and under $\tau \mapsto \tau+1$. We can then write $f\left(z_{1}, z_{2}, \ldots, z_{o} ; \tau\right)=\bar{f}\left(\zeta_{1}, \ldots, \zeta_{n}, q\right)$ where $\zeta_{j}=e^{2 \pi i z_{j}}$ as above. For any permutation $\pi \in S_{n}$ we fix the domain

$$
\Omega_{\pi}=\left|q \zeta_{\pi(i+1)}\right|<\left|\zeta_{\pi(i)}\right|<\left|\zeta_{\pi(i+1)}\right|<1 \quad \text { for all } i=1, \ldots, n-1 .
$$

On such a domain the constant term with respect to all the $\zeta_{i}$ is well-defined. It can be expressed as integral

$$
\left[\zeta_{n}^{0}, \ldots, \zeta_{1}^{0}\right]_{\pi} \bar{f}=\frac{1}{(2 \pi i)^{n}} \oint_{\gamma_{n}} \ldots \oint_{\gamma_{1}} f\left(z_{1}, \ldots, z_{n} ; \tau\right) d z_{1} \ldots d z_{n}
$$

along the integration paths

$$
\gamma_{j}:[0,1] \rightarrow \mathbb{C}, \quad t \mapsto i y_{j}+t,
$$

where $0 \leq y_{\pi(1)}<y_{\pi(2)}<\ldots y_{\pi(n)}<1$. We call these our standard integration paths for the permutation $\pi$. If the domain $\Omega_{\pi}$ is clear from the context we also write $\left[\zeta^{0}\right]$ or $\left[\zeta_{n}^{0}, \ldots, \zeta_{1}^{0}\right]$ as shorthand for the coefficient extraction $\left[\zeta_{n}^{0}, \ldots, \zeta_{1}^{0}\right]_{\pi}$.

Our aim here is to show Theorem 5.8 that for a large class of functions that are quasimodular and quasi-elliptic in $\left(z_{1}, \ldots, z_{2}\right)$ (in a sense made precise below) the constant term with respect to the $\zeta_{i}$ is a quasimodular form.

We start with a preliminary definition of a ring of multi-variable Jacobi forms. For $n \geq 0$ we let $\mathcal{J}_{n}^{(k)}$ be the vector space of meromorphic functions $f$ on $\mathbb{C}^{n} \times \mathbb{H}$ in the variables $\left(z_{1}, \ldots, z_{n} ; \tau\right)$ that

i) have poles on $\mathbb{C}^{n}$ at most at the $(\mathbb{Z}+\tau \mathbb{Z})$-translates of the diagonals $z_{i}-z_{j}$,

ii) are elliptic with respect to the lattice $\mathbb{Z}+\tau \mathbb{Z}$ in the variables $z_{i}$ for $i=$ $1, \ldots, n$, and

iii) are quasi-modular of weight $k$ for $\operatorname{SL}(2, \mathbb{Z})$, i.e. $f$ is holomorphic in $\tau$ on $\mathbb{H} \cup$ $\infty$ and there exists some $p \geq 0$ (called depth) and functions $f_{i}\left(z_{1}, \ldots, z_{n} ; \tau\right)$ that are holomorphic in $\tau$ and meromorphic in the $z_{i}$ such that

$$
(c \tau+d)^{-k} f\left(\frac{z_{1}}{c \tau+d}, \ldots, \frac{z_{n}}{c \tau+d} ; \frac{a \tau+b}{c \tau+d}\right)=\sum_{i=0}^{p} f_{i}\left(z_{1}, \ldots, z_{n} ; \tau\right)\left(\frac{c}{c \tau+d}\right)^{i}
$$

for all $\left(\begin{array}{ll}a & b \\ c & d\end{array}\right) \in \mathrm{SL}(2, \mathbb{Z})$.

Again, taking the identity matrix in this definition implies that $f_{0}=f$.

Proposition 5.3. The direct sum

$$
\mathcal{J}_{n}=\oplus_{k \geq 0} \mathcal{J}_{n}^{(k)}
$$

is a graded ring. The derivatives $\partial / \partial z_{i}$ map $\mathcal{J}_{n}^{(k)}$ to $\mathcal{J}_{n}^{(k+1)}$ for all $i=1, \ldots, n$ and the derivative $D_{q}=q \frac{\partial}{\partial q}$ maps $\mathcal{J}_{n}^{(k)}$ to $\mathcal{J}_{n}^{(k+2)}$.

The $m$-th derivative of the propagator $P^{(m)}\left(z_{i}-z_{j}\right)$ lies in the graded piece $\mathcal{J}_{n}^{(m+2)}$ of weight $m+2$. Moreover, $Z_{i j n}=Z\left(z_{n}-z_{i}\right)+Z\left(z_{j}-z_{n}\right)+Z\left(z_{i}-z_{j}\right)$ lies in the graded piece $\mathcal{J}_{n}^{(1)}$ of weight one. 
Proof. The first two statements obviously follow from the definition and differentiation of the quasimodular transformation property. For the third statement all but the quasimodularity are well-known (and follow also from Theorem [5.2). The quasimodular transformation property iii) follows from (39) using $P_{1}\left(z_{1}, \ldots, z_{n} ; \tau\right)=1$ and $P_{0}=P$. For the last statement, the ellipticity of $Z_{i j n}$ follows from the properties

$$
Z(z+1)=Z(z) \text { and } \quad Z(z+\tau)=Z(z)+1
$$

of the individual summands. Equation (39) again implies that $Z\left(z_{i}-z_{j}\right)$ is quasimodular (in the sense of iii)) of weight one and depth one with $Z_{i}\left(z_{i}-z_{j}\right)=z_{i}-z_{j}$. This in turn implies that $Z_{i j n}$ is even quasimodular of weight one and depth zero, i.e. modular.

Proposition 5.4. For $n=0$ and $n=1$ the ring $\mathcal{J}_{n}$ consists only of the quasimodular forms in $\tau$.

For $n \geq 2$ the ring $\mathcal{J}_{n}$ is generated as a $\mathcal{J}_{n-1}$-module by the derivatives of the $P$-function $P_{j}^{(m)}=P^{(m)}\left(z_{n}-z_{j}\right)$ for all $m \geq 0$ and all $j=1, \ldots, n-1$ and by the linear combinations $Z_{i j n}=Z\left(z_{n}-z_{i}\right)+Z\left(z_{j}-z_{n}\right)+Z\left(z_{i}-z_{j}\right)$ for all $1 \leq i<j \leq n-1$.

More precisely, if $f \in \mathcal{J}_{n}^{(k)}$ then we can write

$$
f=\sum a_{m, j} P_{j}^{(m)}+\sum b_{i, j} Z_{i j n}+c
$$

with $a_{m, j} \in \mathcal{J}_{n-1}^{(k-m-2)}, b_{i, j} \in \mathcal{J}_{n-1}^{(k-1)}$ and $c \in \mathcal{J}_{n-1}^{(k)}$.

Proof. For the first statement we simply note that an elliptic function without poles is constant. For the second statement we argue by induction on the pole orders and we may assume that $f$ is homogeneous of weight $k$. Suppose that $f$ has a pole of order $m$ exactly at $z_{n}-z_{j}$. Then the limit

$$
f^{[j]}\left(z_{1}, \ldots, z_{n-1}\right)=\lim _{\varepsilon \rightarrow 0} \varepsilon^{m} f\left(z_{1}, \ldots, z_{j}, \ldots, z_{n-1}, z_{j}+\varepsilon\right)
$$

exists and is non-zero. We claim that $f^{[j]} \in \mathcal{J}_{n-1}^{(k-m)}$. Conditions i) and ii) are obvious from the definition. If $f_{i}$ denotes a component of $f$ in the modular transformation iii), we define $f_{i}^{[j]}\left(z_{1}, \ldots, z_{n-1}\right)$ in the same way as above for $f=f_{0}$. This transformation implies

$$
\begin{aligned}
& (c \tau+d)^{-k+m}\left(\frac{\varepsilon}{c \tau+d}\right)^{m} f\left(\frac{z_{1}}{c \tau+d}, \ldots, \frac{z_{n-1}}{c \tau+d}, \frac{z_{j}+\varepsilon}{c \tau+d} ; \frac{a \tau+b}{c \tau+d}\right) \\
= & \varepsilon^{m} \sum_{i=0}^{d} f_{i}\left(z_{1}, \ldots, z_{n-1}, z_{j}+\varepsilon ; \tau\right)\left(\frac{c}{c \tau+d}\right)^{i},
\end{aligned}
$$

and taking the limit $\varepsilon \rightarrow 0$ gives the quasimodularity of $f^{[j]}$.

If $m \geq 2$ then replacing $f$ by $f-f^{[j]} P_{j}^{(m-2)}(-2 \pi i)^{m} /(m-1)$ ! decreases the pole order along $z_{n}=z_{j}$ and does not increase the pole order along any of the divisors $z_{n}=z_{i}$ for $i \geq 2$.

Inductively we may thus suppose that $f$ has at most simple poles along all the divisors $z_{n}-z_{j}$. The residue theorem (for $f$ considered as function in $z_{n}$ ) implies 
that $\sum_{j=1}^{n-1} f^{[j]}=0$. Consequently,

$$
g=f-\sum_{j=2}^{n-1}\left(\sum_{i=1}^{j-1} f^{[i]}\right) Z_{j-1, j, n}
$$

is still elliptic and has no poles (considered as function in $z_{n}$ ). This implies that $g \in \mathcal{J}_{n-1}^{(k)}$ and completes the inductive argument.

One is tempted to deduce from this that if $f \in \mathcal{J}_{n}^{k}$ then for any permutation $\pi$ the constant term on $\Omega_{\pi}$ is a quasimodular form of pure weight $k$. This, however is not true! In general, those constant terms are of mixed weight $\leq k$, as we will see in the example in Section 5.4. Even if one is only interested in computing the constant terms of elements in the ring $\mathcal{J}_{n}^{(k)}$, the fact that

$$
\left[\zeta_{n}^{0}\right] P^{(m)}\left(z_{n}-z_{j}\right)=0 \quad(m \geq 0)
$$

but

$$
\left[\zeta_{n}^{0}\right] Z_{i j n}=Z\left(z_{i}-z_{j}\right)
$$

is no longer elliptic forces the consideration of the following more general case of quasi-elliptic functions.

Let $\Delta=\Delta_{\tau}$ be the operator on meromorphic functions defined by

$$
\Delta(f)(z)=f(z+\tau)-f(z) .
$$

A meromorphic function $f$ is called quasi-elliptic if $f(z+1)=f(z)$ and if there exists some positive integer $e$ such that $\Delta^{e}(f)$ is elliptic. The minimal such $e$ is called the order (of quasi-ellipticity) of $f$.

We say that a meromorphic function $f: \mathbb{C}^{n} \times \mathbb{H} \rightarrow \mathbb{C}$ is quasi-elliptic, if it is quasielliptic in each of the first $n$ variables. For such a function we write $\mathbf{e}=\left(e_{1}, \ldots, e_{n}\right)$ for the tuple of orders of quasi-ellipticity in the $n$ variables. Consequently, a quasielliptic function of order $(0, \ldots, 0)$ is simply an elliptic function.

We write $\Delta_{i}$ for the operator $\Delta$ acting on the $i$-th variable. Note that these operators $\Delta_{i}$ commute. We now state the appropriate generalization of the definition of $\mathcal{J}_{n}^{(k)}$ above.

Definition 5.5. We define for $n \geq 0, k \geq 0$ and $\mathbf{e} \geq 0$ the vector space $\mathcal{Q}_{n, \mathbf{e}}^{(k)}$ of quasi-elliptic quasimodular forms to be the space of meromorphic functions $f$ on $\mathbb{C}^{n} \times \mathbb{H}$ in the variables $\left(z_{1}, \ldots, z_{n} ; \tau\right)$ that

i) have poles on $\mathbb{C}^{n}$ at most at the $\mathbb{Z}+\tau \mathbb{Z}$-translates of the diagonals $z_{i}-z_{j}$,

ii) are quasi-elliptic of order $\mathbf{e}$, and

iii) are quasimodular of weight $k$ for $\operatorname{SL}(2, \mathbb{Z})$, i.e. $f$ is holomorphic in $\tau$ on $\mathbb{H} \cup$ $\infty$ and there exists some $p \geq 0$ (called depth) and functions $f_{i}\left(z_{1}, \ldots, z_{n} ; \tau\right)$ that are holomorphic in $\tau$ and meromorphic in the $z_{i}$ such that

$$
(c \tau+d)^{-k} f\left(\frac{z_{1}}{c \tau+d}, \ldots, \frac{z_{n}}{c \tau+d} ; \frac{a \tau+b}{c \tau+d}\right)=\sum_{i=0}^{p} f_{i}\left(z_{1}, \ldots, z_{n} ; \tau\right)\left(\frac{c}{c \tau+d}\right)^{i}
$$

for all $\left(\begin{array}{ll}a & b \\ c & d\end{array}\right) \in \mathrm{SL}(2, \mathbb{Z})$. 
We remark that, contrary to the case of (usual) quasimodular forms, the functions $f_{i}$ do not need to belong to any of the spaces $\mathcal{Q}_{n, \mathbf{e}}^{(k)}$. 3

Proposition 5.6. The direct sum

$$
\mathcal{Q}_{n}=\bigoplus_{k \geq 0} \mathcal{Q}_{n}^{(k)}, \quad \text { where } \mathcal{Q}_{n}^{(k)}=\bigoplus_{\mathbf{e} \geq 0} \mathcal{Q}_{n, \mathbf{e}}^{(k)},
$$

is a graded ring. The derivatives $\partial / \partial z_{i}$ map $\mathcal{Q}_{n}^{(k)}$ to $\mathcal{Q}_{n}^{(k+1)}$ for all $i=1, \ldots, n$. and the derivative $D_{q}=q \frac{\partial}{\partial q}$ maps $\mathcal{Q}_{n}^{(k)}$ to $\mathcal{Q}_{n}^{(k+2)}$.

For all $i, j \in\{1, \ldots, n\}$ the functions

$$
L\left(z_{i}-z_{j}\right)=-\frac{1}{2} Z^{2}\left(z_{i}-z_{j}\right)+\frac{1}{2} P\left(z_{i}-z_{j}\right)-G_{2}+\frac{1}{12}
$$

belong to $\mathcal{Q}_{n}^{(0)} \oplus \mathcal{Q}_{n}^{(2)}$.

Proof. For the proof that $\mathcal{Q}_{n}$ is closed under multiplication we remark that $\Delta_{i}^{m}(f g)$ is a sum of products of $\Delta_{i}^{m_{1}}(f)$ and $\Delta_{i}^{m_{2}}(g)$ with $m_{1}+m_{2}=m$, evaluated at arguments translated by multiples of $\tau$. Consequently, if $f \in \mathcal{Q}_{n, \mathbf{e}_{1}}^{\left(k_{1}\right)}$ and $g \in \mathcal{Q}_{n, \mathbf{e}_{2}}^{\left(k_{2}\right)}$, then $f g \in \mathcal{Q}_{n, \mathbf{e}_{1}+\mathbf{e}_{2}}^{\left(k_{1}+k_{2}\right)}$. The property of the derivatives can be checked as in the case of $\mathcal{J}_{n}$.

For the last identity we use the elliptic transformation law (38) to deduce that $L+\frac{Z^{2}}{2}$ is elliptic. The first terms of the Laurent series of this function are

$$
L(z)+\frac{Z^{2}(z)}{2}=\frac{1}{2 u^{2}}+\frac{1}{12}+O\left(z^{2}\right) .
$$

Consequently, $L+\frac{Z^{2}}{2}-\left(\frac{1}{2} P-G_{2}+\frac{1}{12}\right)$ is an elliptic function with no poles, i.e. it is constant with respect to $z$. Moreover this constant is the value at $z=0$, which is 0 .

Proposition 5.7. The vector space $\mathcal{Q}_{n}$ is (additively) generated as $\mathcal{Q}_{n-1}$-module by the functions $Z^{e}\left(z_{n}-z_{j}\right) P^{(m)}\left(z_{n}-z_{j}\right)$ for $j=1, \ldots, n-1$ and for all $e \geq 0$ and $m \geq 0$.

More precisely, if $f \in \mathcal{Q}_{n}^{(k)}$ then we can write

$$
f\left(z_{1}, \ldots, z_{n}\right)=\sum_{e, m, j} a_{e, m, j} Z^{e}\left(z_{n}-z_{j}\right) P^{(m)}\left(z_{n}-z_{j}\right)+\sum_{e, j} b_{e, j} Z^{e}\left(z_{n}-z_{j}\right)+c
$$

with $a_{e, m, j} \in \mathcal{Q}_{n-1}^{(k-e-m-2)}, b_{e, j} \in \mathcal{Q}_{n-1}^{(k-e)}$ and $c \in \mathcal{Q}_{n-1}^{(k)}$.

Proof. For every $n$ we argue inductively on the order $e=\min _{j \geq 0}\left\{\Delta_{n}^{j}(f)\right.$ elliptic $\}$ of quasi-ellipticity with respect to the last variable. Suppose, without loss of generality, that $f \in \mathcal{Q}_{n}^{(k)}$ is homogeneous of weight $k$. If $e=0$ then we proceed as in the proof of Proposition 5.4 and subtract $\mathcal{Q}_{n-1}$-multiples of $P^{(m)}\left(z_{n}-z_{j}\right)$ for appropriate $m$ and $j \in\{1, \ldots, n-1\}$ and finally $\mathcal{Q}_{n-1}$-multiples of $Z\left(z_{n}-z_{j}\right)-Z\left(z_{n}-z_{k}\right)$

${ }^{3}$ For example, already for $Z\left(z_{i}-z_{j}\right)$ we have seen $Z_{1}=z_{i}-z_{j}$, which is not 1-periodic. Our definition that imposes 1 -periodicity (and thus breaks the symmetry in the role of the $\mathbb{Z}+\tau \mathbb{Z}$ lattice) is the reason for this. A more conceptual definition would be to consider functions that are annihilated by some power of both shift operators $\Delta_{\tau}$ and $\Delta_{1}$ with respect to every variable. E.g. the function $z_{i}-z_{j}$ has this property. Since we do not aim e.g. for an operation of $\mathfrak{s l}(2)$ on our ring of quasi-elliptic quasimodular forms, we will not discuss this generalization here. 
so that the resulting function is elliptic and without poles in the variable $z_{n}$, hence constant in $z_{n}$.

Now consider the case $e>0$. Using the induction hypothesis we can write

$$
\Delta_{n}(f)\left(z_{1}, \ldots, z_{n}\right)=\sum_{s=0}^{e-1} \sum_{j, m} a_{s, j, m} Z^{s}\left(z_{n}-z_{j}\right) P^{(m)}\left(z_{n}-z_{j}\right)+b_{s, j} Z^{s}\left(z_{n}-z_{j}\right) .
$$

Now set

$\tilde{f}\left(z_{1}, \ldots, z_{n}\right)=\sum_{s=0}^{e-1} \frac{1}{s+1} \sum_{j, m} a_{s, j, m} Z^{s+1}\left(z_{n}-z_{j}\right) P^{(m)}\left(z_{n}-z_{j}\right)+b_{s, j} Z^{s}\left(z_{n}-z_{j}\right)$.

Then, since

$$
\Delta_{n}\left(Z^{s+1}\left(z_{n}\right) P^{(m)}\left(z_{n}\right)\right)=P^{(m)}\left(z_{n}\right) \sum_{t=0}^{s}\left(\begin{array}{c}
s+1 \\
t
\end{array}\right) Z^{t}\left(z_{n}\right)
$$

we conclude that

$$
\begin{gathered}
\Delta_{n}(f-\tilde{f})\left(z_{1}, \ldots, z_{n}\right)=\sum_{s=0}^{e-1} \frac{1}{s+1}\left(\sum_{j, m} a_{s, j, m} P^{(m)}\left(z_{n}-z_{j}\right)+b_{s, j}\right) \\
\cdot\left(\sum_{t=0}^{s-1}\left(\begin{array}{c}
s+1 \\
t
\end{array}\right) Z^{t}\left(z_{n}-z_{j}\right)\right)
\end{gathered}
$$

has order $\leq e-2$ with respect to $z_{n}$. Consequently, $f-\tilde{f}$ has order $\leq e-1$ and another application of the induction hypothesis implies the claim.

Using this additive basis we can now prove the main result, that contains Theorem 1.1 as special case.

Theorem 5.8. For any permutation $\pi$ the constant term with respect to the domain $\Omega_{\pi}$ of a function in $\mathcal{Q}_{n}^{(k)}$ is a quasimodular form of mixed weight $\leq k$.

Proof. By relabeling the variables of $f \in \mathcal{Q}_{n}^{(k)}$ we may assume that $\pi$ is the trivial permutation. We may thus write $f$ as in Proposition 5.7 and integrate with respect to $z_{n}$ first. We are thus reduced to showing that the $z_{n}$-integrals of the additive generators $Z^{e}\left(z_{n}-z_{j}\right) P^{(m)}\left(z_{n}-z_{j}\right)$ and $Z^{e}\left(z_{n}-z_{j}\right)$ are quasimodular forms of mixed weight less or equal to $e+2+m$. For the generators including a $P$-derivative we use the integration by parts $\left[Z^{e}(z)\right]^{\prime}=e Z^{e-1}(z) P(z)$ and

$$
\left[Z^{e}(z) P^{(m-1)}(z)\right]^{\prime}=e Z^{e-1}(z) P(z) P^{(m-1)}(z)+Z^{e}(z) P^{(m)}(z)
$$

to reduce the order $e$ until we can apply (45). This involves rewriting $P(z) P^{(m-1)}(z)$ as a linear combination of $P^{(j)}(z)$. To do this, we use again Proposition 5.4 and note that the terms $Z_{i j n}$ will not occur in this case, since the expression $P(z) P^{(m-1)}(z)$ has a unique pole at $z=0$ modulo $\mathbb{Z}+\tau \mathbb{Z}$, and hence zero residue there. The integrals of the remaining generators are dealt with in the next proposition.

In all the steps so far the weight of the quasimodular form had been preserved. We isolate the next step since this is the reason for mixed weight.

Proposition 5.9. The constant coefficient $\left[\zeta^{0}\right] Z^{e}(z)$ is a quasimodular form of mixed weight less or equal to $e$. 
Proof. Since $Z(z)$ is an odd function of $z$, we obtain for $\ell$ odd

$$
\begin{aligned}
\int_{0}^{1} Z^{\ell}(z+i \varepsilon) d z & =\int_{-1 / 2}^{1 / 2} Z^{\ell}(z+i \varepsilon) d z=-\int_{-1 / 2}^{1 / 2} Z^{\ell}(z-i \varepsilon) d z \\
& =-\frac{1}{2} \operatorname{Res}_{0} Z^{\ell}
\end{aligned}
$$

and these residues can be read off from the Laurent series development (42) raised to the $\ell$-th power.

On the other hand, we claim that $\left[\zeta^{0}\right](Z-1 / 2)^{\ell}=0$ for $\ell$ odd. To see this, we expand the product of the Fourier expansions (41) to obtain

$$
(Z-1 / 2)^{\ell}=\sum_{\substack{k_{1}, \ldots, k_{\ell} \geq 1 \\ \varepsilon_{1}, \ldots, \varepsilon_{n} \in\{ \pm 1\}}}(-1)^{\sum \varepsilon_{i}} \prod_{i=1}^{\ell} \frac{Q_{i}\left(k_{i}, \varepsilon_{i}\right)}{1-q^{k_{i}}},
$$

where $Q_{i}\left(k_{i}, \varepsilon_{i}\right)=1$ for $\varepsilon=1$ and $Q_{i}\left(k_{i}, \varepsilon_{i}\right)=q^{k_{i}}$ for $\varepsilon=-1$. The constant term $\left[\zeta^{0}\right](Z-1 / 2)^{\ell}$ is equal to the sum over all $k_{i}$ and $\varepsilon_{i}$ with $\sum k_{i} \varepsilon_{i}=0$ of the right hand side of (48). This set admits and involution by swapping the signs of all the $\varepsilon_{i}$. This involutions changes the sign of the prefactor $(-1)^{\sum \varepsilon_{i}}$ since $\ell$ is odd. We claim that the product is unchanged by the involution. The denominator obviously does not change. The numerator is $q$ raised to the power $\sum_{i: \varepsilon_{i}=+1} k_{i}$ in one case while it is $q$ raised to the power $\sum_{i: \varepsilon_{i}=-1} k_{i}$ in the other case. The two sums are equal by the defining condition of the constant term.

Knowing the constant terms of $(Z-1 / 2)^{\ell}$ and $Z^{\ell}$ for $\ell$ odd, we can solve the (triangular) system of linear equations to determine the constant coefficients of $Z^{\ell}$ for $\ell$ even.

The first few values of these constant coefficients of $Z^{\ell}$ are

$$
\begin{aligned}
{\left[\zeta^{0}\right] Z } & =\frac{1}{2} & {\left[\zeta^{0}\right] Z^{2} } & =-2 G_{2}+\frac{1}{6} \\
{\left[\zeta^{0}\right] Z^{3} } & =-3 G_{2} & {\left[\zeta^{0}\right] Z^{4} } & =8 G_{2}^{2}-\frac{1}{3} G_{4}-2 G_{2}-\frac{1}{30} \\
{\left[\zeta^{0}\right] Z^{5} } & =20 G_{2}^{2}-\frac{5}{6} G_{4} & {\left[\zeta^{0}\right] Z^{6} } & =\frac{-1}{60} G_{6}+4 G_{4} G_{2}-40 G_{2}^{3}+20 G_{2}^{2}-\frac{5}{6} G_{4}+G_{2}+\frac{1}{42}
\end{aligned}
$$

5.4. An example of mixed weight. Here we illustrate that the proof of Theorem 5.8 provides an effective algorithm by computing (with respect to the standard order (43)) the expression

$$
\begin{aligned}
& {\left[\zeta^{0}\right] P\left(z_{1}-z_{2}\right)^{2} P\left(z_{1}-z_{4}\right) P\left(z_{2}-z_{3}\right) P\left(z_{3}-z_{4}\right)^{2} } \\
= & 4 q^{2}+224 q^{3}+3088 q^{4}+21888 q^{5}+105136 q^{6}+388288 q^{7}+1197280 q^{8}+O\left(q^{9}\right) \\
= & -256 G_{2}^{6}+\frac{640}{3} G_{4} G_{2}^{4}+\frac{112}{9} G_{6} G_{2}^{3}-\frac{400}{9} G_{4}^{2} G_{2}^{2}-\frac{140}{9} G_{6} G_{4} G_{2}+\frac{2000}{81} G_{4}^{3}+\frac{49}{108} G_{6}^{2} \\
& +\left(-\frac{256}{3} G_{4} G_{2}^{3}-\frac{16}{5} G_{6} G_{2}^{2}+\frac{320}{21} G_{4}^{2} G_{2}+\frac{28}{9} G_{6} G_{4}\right),
\end{aligned}
$$

which is a quasimodular form of mixed weight 10 and 12 . 
To prove this formula we first treat the terms depending on $z_{4}$ and write

$$
\begin{aligned}
P\left(z_{4}-z_{1}\right)^{2} P\left(z_{4}-z_{3}\right) & =\frac{1}{6} P\left(z_{1}-z_{3}\right) P^{\prime \prime}\left(z_{4}-z_{1}\right)+\frac{1}{2} P^{\prime}\left(z_{3}-z_{1}\right) P^{\prime}\left(z_{4}-z_{1}\right) \\
& +\left(\frac{1}{2} P^{\prime \prime}\left(z_{1}-z_{3}\right)+4 G_{2} P\left(z_{1}-z_{3}\right)\right) P\left(z_{4}-z_{1}\right) \\
& +P\left(z_{1}-z_{3}\right)^{2} P\left(z_{4}-z_{3}\right) \\
& +\left(4 G_{2} P^{\prime}\left(z_{3}-z_{1}\right)-\frac{1}{6} P^{\prime \prime \prime}\left(z_{1}-z_{3}\right)\right) Z_{134} \\
& +\frac{1}{3} G_{2} P^{\prime \prime}\left(z_{1}-z_{3}\right)-2 G_{2} P\left(z_{1}-z_{3}\right)^{2}-8 G_{2}^{2} P\left(z_{1}-z_{3}\right) \\
& +8 G_{2}^{3}-\frac{10}{3} G_{4} G_{2}
\end{aligned}
$$

in the additive basis given in Proposition 5.7. This allows to integrate with respect to $z_{4}$ and we obtain

$$
\begin{aligned}
{\left[\zeta_{4}^{0}\right] P\left(z_{4}-z_{1}\right)^{2} P\left(z_{4}-z_{3}\right)=} & \left(4 G_{2} P^{\prime}\left(z_{3}-z_{1}\right)-\frac{1}{6} P^{\prime \prime \prime}\left(z_{3}-z_{1}\right)\right) Z\left(z_{1}-z_{3}\right) \\
& +\frac{4}{3} G_{2} P^{\prime \prime}\left(z_{3}-z_{1}\right)+8 G_{2}^{3}-\frac{10}{3} G_{4} G_{2} .
\end{aligned}
$$

The integral $\left[\zeta_{2}^{0}\right] P\left(z_{1}-z_{2}\right) P\left(z_{2}-z_{3}\right)^{2}$ is the same as the previous one, replacing $z_{4}$ by $z_{2}$. The product of these two integrals contains the term

$$
T=P^{\prime \prime \prime}\left(z_{1}-z_{3}\right) P^{\prime}\left(z_{1}-z_{3}\right) Z\left(z_{1}-z_{3}\right)^{2}
$$

and several terms that can be treated similarly as $T$, or that can be computed by integration by parts as in the proof of Theorem 5.8 and that finally yield a contribution that is pure of weight 12 . To integrate $T$ with respect to $z_{1}$ we decompose $T / Z\left(z_{1}-z_{3}\right)^{2}$ in the additive basis

$$
P^{\prime \prime \prime}(z) P^{\prime}(z)=-12 G_{6} P(z)+24 G_{6} G_{2}-\frac{800}{7} G_{4}^{2}-8 P^{\prime \prime}(z) G_{4}+\frac{1}{105} P^{(6)}(z)
$$

given by Proposition 5.4 The $Z^{2}$-multiples of the terms containing $P$ or its derivatives can be computed using integration by parts and contribute purely to weight 12 . Finally, the constant (in $z$ ) multiples of $Z^{2}$ are integrated using Proposition 5.9 and cause the contribution of mixed weight.

\section{Quasimodularity of Graph Sums}

Motivated by Theorem 4.3 and 4.1 we consider here the graph sums

$$
S(\Gamma, \mathbf{m})=\sum_{G \in \Gamma} S(G, \mathbf{m})
$$

over all orientations $G$ of $\Gamma$, where

$$
S(G, \mathbf{m})=\sum_{h \in \widetilde{\mathbb{N}}^{E(G)}, w \in \mathbb{Z}_{+}^{E(G)}} \prod_{i \in E(G)} w_{i}^{m_{i}+1} q^{h_{i} w_{i}} \prod_{v \in V(G)} \delta(v) .
$$

Here $\widetilde{\mathbb{N}}^{E(G)}$ is the height space introduced in (18) and the Dirac-symbol $\delta(v)$ was introduced in (29). The goal of this section is to show the quasimodularity of these graph sums.

Theorem 6.1. If $\mathbf{m}=\left(m_{1}, \ldots, m_{|E(\Gamma)|}\right)$ is a tuple of even integers, then the graph sums $S(\Gamma, \mathbf{m})$ are quasimodular forms of mixed weight at most $k(\mathbf{m}):=\sum_{i}\left(m_{i}+2\right)$. 
The proof consists of splitting the sum into the contribution from loops and the rest, and then to apply the coefficient extraction results from Section 5.1 .

The combination of this result with the graph sum theorem and the polynomiality of generalized double Hurwitz numbers with completed cycles immediately gives the first quasimodularity result we are aiming for.

Corollary 6.2. For any ramification profile $\Pi$ the counting function $N^{\circ}(\Pi)$ for connected torus covers of profile $\Pi$ is a quasimodular form of mixed weight less or equal to $\mathrm{wt}(\Pi)=|\Pi|+\ell(\Pi)$.

Proof. As remarked before Theorem 4.3. thanks to Theorem 3.1 we only need to show the quasimodularity of $q$-brackets of a product of $p_{\ell_{i}}, i=1 \ldots n$, i.e. of the expressions appearing in (31) that are sums over graphs with $n$ labeled vertices. By Theorem 4.1 the expressions $\bar{A}_{v}^{\prime}=\bar{A}^{\prime}\left(\mathbf{w}_{v}^{-}, \mathbf{w}_{v}^{+},\left(\ell_{\# v}\right)\right)$ appearing on the right hand side of (31) are either zero or they are polynomials of even degree equal to $\ell_{\# v}+1-\ell\left(\mathbf{w}_{v}^{-}\right)-\ell\left(\mathbf{w}_{v}^{+}\right)$. Consequently, the product over all vertices of these polynomials $\bar{A}_{v}^{\prime}$ has degree $\operatorname{wt}(\Pi)-2|E(\Gamma)|$. We can now apply Theorem 6.1 .

The rest of this section consists of the proof of Theorem 6.1 As a first technical step we show that $S(\Gamma, \mathbf{m})$, which a priori depends on the heights $y_{i}$ involved in the definition of the height space, is in fact independent of this choice and can be computed using the limit value $y_{i}=0$ for all $i$.

Lemma 6.3. Replacing the height space $\widetilde{\mathbb{N}} E(G)$ by

$$
\mathbb{N}_{E(G)}=\left\{\left(n_{1}, \ldots, n_{|E(G)|}\right), n_{i} \in\{0\} \cup \mathbb{Z}_{+} \text {if } v_{+}(i)>v_{-}(i), n_{i} \in \mathbb{Z}_{+} \text {otherwise }\right\}
$$

does not change the total sum $S(G, \mathbf{m})$.

Proof. We apply the linear change of variables $h_{e}^{\prime}=h_{e}-\delta_{e}$ with $\delta_{e}=\varepsilon_{i+(e)}-\varepsilon_{i^{-}(e)}$, that maps $\widetilde{\mathbb{N}}^{E(G)}$ onto $\mathbb{N}_{E(G)}$. Each summand for fixed $\left(w_{1}, \ldots, w_{|E(G)|}\right)$ is then multiplied by

$$
q^{-\sum_{i} \delta_{i} w_{i}} \prod_{v \in V(G)} \delta(v)=q^{-\frac{1}{2} \sum_{v} \varepsilon_{v}\left(\sum_{i \in e_{-}(v)} w_{i}-\sum_{i \in e_{+}(v)} w_{i}\right)} \prod_{v \in V(G)} \delta(v)=1
$$

This implies the claim.

6.1. The reduced graph. We call an edge of a graph $\Gamma$ (or an oriented graph $G$ ) neutral, if it is a loop (starting and ending at the same vertex). The other edges are called essential. We denote by $E(G)=E_{0}(G) \cup E_{1}(G)$ the splitting of the set of edges of $G$ into neutral and essential edges. Furthermore, let $G_{1}$ denote the oriented graph obtained from $G$ by removing the neutral edges. Similarly, $\Gamma_{1}$ is the graph obtained from $\Gamma$ by removing the neutral edges. A graph with no neutral edges we be called reduced.

Lemma 6.4. The graph sums $S(\Gamma, \mathbf{m})$ can be factored as

$$
S(\Gamma, \mathbf{m})=S_{0}\left(\Gamma, \mathbf{m}_{0}\right) S\left(\Gamma_{1}, \mathbf{m}_{1}\right)
$$

where

$$
S_{0}\left(\Gamma, \mathbf{m}_{0}\right)=\sum_{h \in \mathbb{N}_{E_{0}(G)}, w \in \mathbb{Z}_{+}^{E_{0}(G)}} \prod_{i \in E_{0}(G)} w_{i}^{m_{i}+1} q^{h_{i} w_{i}},
$$

for any orientation $G$ of $\Gamma$, and where $\mathbf{m}_{0}=\left(m_{i}\right)_{i \in E_{0}(G)}, \mathbf{m}_{1}=\left(m_{i}\right)_{i \in E_{1}(G)}$. 
Proof. This follows from decomposing for every $G \in \Gamma$ the summation in the definition of $S(G, \mathbf{m})$ into the sum over $h \in \mathbb{N}_{E_{0}(G)}$ and $w \in \mathbb{Z}_{+}^{E_{0}(G)}$ and the remaining variables. Since the $w_{i}$ for $i \in E_{0}(G)$ drop out of the functions $\delta$, each $S(G, \mathbf{m})$ splits off a factor

$$
S_{0}\left(G, \mathbf{m}_{0}\right)=\sum_{h \in \mathbb{N}_{E_{0}(G)}, w \in \mathbb{Z}_{+}^{E_{0}(G)}} \prod_{i \in E_{0}(G)} w_{i}^{m_{i}+1} q^{h_{i} w_{i}}
$$

which does not depend of the orientation $G$ of $\Gamma$.

Lemma 6.5. If $\mathbf{m}=\left(m_{1}, \ldots, m_{|E(G)|}\right)$ is a tuple of even integers, the neutral contribution $S_{0}\left(\Gamma, \mathbf{m}_{0}\right)$ is a quasimodular form of mixed weight $k\left(\mathbf{m}_{0}\right)$.

Proof. The neutral contribution $S_{0}\left(\Gamma, \mathbf{m}_{0}\right)$ is the product of $S_{m_{i}}$ over all neutral edges, where $S_{m}=\sum_{w, h=1}^{\infty} w^{m+1} q^{h w}$ is the $q$-expansion of the Eisenstein series $G_{m+2}$ without its constant term for $m$ even.

The problem is now reduced to computing $S(\Gamma, \mathbf{m})$ for all reduced graphs $\Gamma$.

6.2. Contour integrals. The idea of the proof is to write the delta functions appearing in the graph sums as contour integral of some suitably chosen powers of $\zeta=e^{2 \pi i z}$ since by the residue theorem

$$
\int_{\gamma} \zeta^{w} d z=\int_{\gamma^{\prime}} \zeta^{w-1} \frac{d \zeta}{2 \pi i}=\delta_{0, w}
$$

where $\gamma(t)=t+i y$ with $0 \leq t \leq 1$ is our standard integration path of height $\Im y \in(0, \Im \tau)$ and $\gamma^{\prime}=\exp (2 \pi i \gamma)$. Recall that we denote the derivative with respect to $u=2 \pi i z$ by primes. By (41) the Fourier developments of the derivatives of $P$ are

$P^{(m)}(z)=\frac{1}{(2 \pi i)^{m}} \frac{\partial^{m}}{\partial z^{m}} P(z)=\sum_{w=1}^{\infty} \sum_{h=1}^{\infty} w^{m+1} q^{h w}\left(\zeta^{w}+(-1)^{m} \zeta^{-w}\right)+\sum_{w=1}^{\infty} w^{m+1} \zeta^{w}$.

Next, we expand the path integrals of products of derivatives of $P$. In the following lemmas $\gamma_{j}$ will be the standard path at height $y_{j}$ and $y_{j}<y_{j+1}$.

Lemma 6.6. Let $I \subset\{2, \ldots, n\}$ and assume that all the $m_{i}$ are even. Then on the domain defined by $\left|\zeta_{i}\right|>\left|\zeta_{1}\right|>\left|q \zeta_{i}\right|$ for all $i \in I$ we have the expansion

$$
\begin{aligned}
\oint_{\gamma_{1}} \zeta_{1}^{k_{1}} \prod_{i \in I} P^{\left(m_{i}\right)}\left(z_{1}-z_{i}\right) d z_{1}= & \sum_{w_{i}=1}^{\infty} \prod_{i \in I} w_{i}^{m_{i}+1} \cdot \sum_{I=J \cup K} \delta\left(k_{1}+\sum_{j \in J} w_{j}-\sum_{k \in K} w_{k}\right) \\
& \cdot \prod_{j \in J}\left(\sum_{h_{j}=0}^{\infty} q^{h_{j} w_{j}}\right) \cdot \prod_{k \in K}\left(\sum_{h_{k}=1}^{\infty} q^{h_{k} w_{k}}\right) \frac{\prod_{K} \zeta_{k}^{w_{k}}}{\prod_{J} \zeta_{j}^{w_{j}}}
\end{aligned}
$$

for every $k_{1} \in \mathbb{Z}$.

Proof. Since $P$ is even by definition, so are its derivatives of even order. The lemma follows by expanding the integrand according to the preceding formula for $P^{(m)}$ and by (52) only the term with $k_{1}+\sum_{j \in J} w_{j}-\sum_{k \in K} w_{k}=0$ survives.

We can use this observation to write the graph sum in terms of derivatives of $P$. For this purpose, we introduce the following shorthand notations. Let $\mathbf{z}=$ 
$\left(z_{1}, \ldots, z_{n}\right)$. For a reduced graph $\Gamma_{1}$ with $n$ vertices and $N_{1}$ edges, for $\left(y_{1}, \ldots, y_{n}\right)$ fixed as before, and $\mathbf{m}_{1}=\left(m_{1}, \ldots, m_{N_{1}}\right)$ an $N_{1}$-tuple of even integers, we define

$$
P_{\Gamma_{1}, \mathbf{m}_{1}}(\mathbf{z})=\prod_{i \in E\left(\Gamma_{1}\right)} P^{\left(m_{i}\right)}\left(z_{v_{1}(i)}-z_{v_{2}(i)}\right),
$$

where $v_{1}(i)$ and $v_{2}(i)$ are the two ends of the edge $i$. Note that by our parity assumption the function $P^{\left(m_{i}\right)}$ is even and so the expression is independent of the labeling of the ends of edges.

Proposition 6.7. For a tuple of even integers $\mathbf{m}_{1}$ we can express the graph sum as

$$
S\left(\Gamma_{1}, \mathbf{m}_{1}\right)=\left[\zeta_{n}^{0}, \ldots, \zeta_{1}^{0}\right] P_{\Gamma_{1}, \mathbf{m}_{1}}(\mathbf{z}),
$$

where the coefficient extraction is for the expansion on the domain $\left|q \zeta_{i+1}\right|<\left|\zeta_{i}\right|<$ $\left|\zeta_{i+1}\right|<1$ for all $i$.

Proof. By the general observation in Section 5.3, coefficient extraction is the same operation as the computation of the path integrals along the standard paths. We compute the right hand side inductively and show that the final expressing coincides with the graph sum. We denote by $E_{1}$ the set of the (labels of the) edges adjacent to the vertex $v_{1}$. For two subsets of edge labels we define the shorthand notation

$$
\delta(J, K)=\delta\left(\sum_{j \in J} w_{j}-\sum_{k \in K} w_{k}\right) .
$$

Using that $\Gamma_{1}$ has no loops and the parity of $P$ we rewrite the integrand as

$$
P_{\Gamma_{1}, \mathbf{m}_{1}}(\mathbf{z})=\prod_{j \in E_{1}} P^{\left(m_{j}\right)}\left(z_{1}-z_{v_{\text {other }}(j)}\right) \cdot \prod_{i \in E \backslash E_{1}} P^{\left(m_{j}\right)}\left(z_{v_{1}(j)}-z_{v_{2}(j)}\right)
$$

where $v_{\text {other }}(j)=v_{o}(j)$ is the second extremity of the edge $j$ for each edge label $j$ adjacent to $v_{1}$. We now apply Lemma 6.6 with $k_{1}=0$ and obtain

$$
\begin{aligned}
& \oint_{\gamma_{1}} P_{\Gamma_{1}, \mathbf{m}_{1}}(\mathbf{z})=\prod_{i \in E \backslash E_{1}} P^{\left(m_{i}\right)}\left(z_{v_{1}(i)}-z_{v_{2}(i)}\right) \cdot \sum_{\substack{w_{i}=1 \\
i \in E_{1}}}^{\infty}\left(\prod_{i \in E_{1}} w_{i}^{m_{i}+1}\right) \\
& \cdot \sum_{E_{1}=J_{1} \sqcup K_{1}} \delta\left(J_{1}, K_{1}\right) \prod_{j \in J_{1}}\left(\sum_{h_{j}=1}^{\infty} q^{h_{j} w_{j}}\right) \prod_{k \in K_{1}}\left(\sum_{h_{k}=0}^{\infty} q^{h_{k} w_{k}}\right) \frac{\prod_{K_{1}} \zeta_{v_{o}(k)}^{w_{k}}}{\prod_{J_{1}} \zeta_{v_{o}(j)}^{w_{j}}}
\end{aligned}
$$

Consider the new graph $\Gamma^{(1)}$ obtained by removing the edges in $E_{1}$ from $\Gamma_{1}$. We denote by $E_{2}$ the set of edges adjacent to $v_{2}$ in this new graph, and for each partition $J_{1} \sqcup K_{1}$ of $E_{1}$ we iterate the process by integrating

$$
\prod_{i \in E \backslash E_{1}} P^{\left(m_{i}\right)}\left(z_{v_{1}(i)}-z_{v_{-}(i)}\right) \cdot \frac{\prod_{K_{1}} \zeta_{v_{o}(k)}^{w_{k}}}{\prod_{J_{1}} \zeta_{v_{o}(j)}^{w_{j}}}
$$

along $\gamma_{2}$, using again Lemma 6.6. We then consider the graph $\Gamma^{(2)}$ obtained by removing the edges in $E_{2}$ from $\Gamma^{(1)}$ and so forth. At the end of this procedure, we 
obtain

$$
\begin{aligned}
& {\left[\zeta_{n}^{0}, \ldots, \zeta_{1}^{0}\right] P_{\Gamma_{1}, \mathbf{m}_{1}}(\mathbf{z}) } \\
= & \sum_{h_{i}, w_{i}} \prod_{i} w_{i}^{m_{i}+1} \cdot \sum_{E_{1}=J_{1} \sqcup K_{1}} \delta\left(J_{1}, K_{1}\right) \cdot \prod_{j \in J_{1}} \sum_{h_{j}=1}^{\infty} q^{h_{j} w_{j}} \cdot \prod_{k \in K_{1}} \sum_{h_{k}=0}^{\infty} q^{h_{k} w_{k}} \\
& \cdot \sum_{E_{2}=J_{2} \sqcup K_{2}} \delta\left(J_{2} \sqcup K_{1}^{2}, K_{2} \sqcup J_{1}^{2}\right) \cdot \prod_{j \in J_{2}} \sum_{h_{j}=1}^{\infty} q^{h_{j} w_{j}} \cdot \prod_{k \in K_{2}} \sum_{h_{k}=0}^{\infty} q^{h_{k} w_{k}} \\
& \ldots \sum_{E_{n}=J_{n} \sqcup K_{n}} \delta\left(J_{n} \sqcup K_{n-1}^{n} \sqcup \ldots, K_{n} \sqcup J_{n-1}^{n} \sqcup \ldots\right) \cdot \prod_{j \in J_{n}} \sum_{h_{j}=1}^{\infty} q^{h_{j} w_{j}} \cdot \prod_{k \in K_{n}} \sum_{h_{k}=0}^{\infty} q^{h_{k} w_{k}}
\end{aligned}
$$

where $K_{i}^{j}$ denotes the subset of $K_{i}$ formed by the edges adjacent to $v_{j}$. We recognize the definition of $S\left(\Gamma_{1}, m\right)$, since partitioning $E$ into $E_{1}, E_{2}, \ldots E_{n}$ as above gives an orientation $G_{1}$ of $\Gamma_{1}$, and non realizable orientations have coefficient 0 . Note that the last integration with respect to the variable $z_{n}$ has no effect since the powers of $\zeta_{n}$ cancel out thanks to the delta functions.

Proof of Theorem 6.1. In view of the factorization of the quantity we are interested in the loop contribution and the reduced contribution in Lemma 6.4. The loop contribution is quasimodular by Lemma 6.5, so it suffices to show that the right hand side of (54) is a quasimodular form of mixed weight $\leq k\left(\mathbf{m}_{1}\right)$. This follows

from Theorem 5.8, since $P_{\Gamma_{1}, \mathbf{m}_{1}} \in \mathcal{J}_{n}^{\left(k\left(\mathbf{m}_{1}\right)\right)} \subset \mathcal{Q}_{n}^{\left(k\left(\mathbf{m}_{1}\right)\right)}$ by Proposition 5.6.

Proof of Theorem 3.2. Let $f$ be a shifted symmetric function of weight $k$. By Theorem 3.1, $f$ is a linear combination of products of $P_{l}$, each product being of weight smaller or equal to $k$. Let $P_{\ell_{1}} \ldots P_{\ell_{n}}$ be such a product. We claim that $\left\langle P_{\ell_{1}} \ldots P_{\ell_{n}}\right\rangle_{q}$ is quasimodular of weight smaller than $k$. By Theorem 4.3, such a term decomposes as a graph sum (31), where the completed Hurwitz numbers $\bar{A}^{\prime}$ that appear in the graph sum are some even polynomials in the $w_{i}$ (Theorem 4.1). The product $\prod_{v \in G} \bar{A}^{\prime}\left(\mathbf{w}_{v}^{-}, \mathbf{w}_{v}^{+},\left(\ell_{\# v}\right)\right)$ is an even polynomial of degree $\leq k-2|E(G)|$. Considering each monomial of degree $\mathbf{m}=\left(m_{1}, \ldots, m_{|E(G)|}\right)$, we get the graph sum $S(G, \mathbf{m})$ in (51), which is quasimodular of weight $|\mathbf{m}|+2|E(G)|$ by Theorem 6.1] By linearity we obtain the quasimodularity of weight $\leq k$ of each $\left\langle P_{\ell_{1}} \ldots P_{\ell_{n}}\right\rangle_{q, G}$, hence the quasimodularity each $\left\langle P_{\ell_{1}} \ldots P_{\ell_{n}}\right\rangle_{q}$, and finally the quasimodularity of the $\langle f\rangle_{q}$.

\section{Siegel-Veech COnstants}

The study of area Siegel-Veech constants for flat surfaces, briefly recalled in Section 7.1, lead in [CMZ16] to counting problems for graphs sums with a Siegel-Veech weight that we introduce in the sequel. We show that the Siegel-Veech weighted graph sums admit a decomposition into graph sums of triple Hurwitz numbers similar to the unweighted case (Proposition 4.2). The main result (Theorem [7.3) in this section is that these graph sums fit into the scope of our quasimodularity machinery of Section 5. We use this to give another proof of the quasimodularity of the Siegel-Veech weighted generating series observed in [CMZ16]. 
Let $\lambda=\left(\lambda_{1} \geq \lambda_{2} \geq \cdots \geq \lambda_{k}\right)$ be a partition. For $p \in \mathbb{Z}$ we define the $p$-th Siegel-Veech weight of $\lambda$ to be

$$
S_{p}(\lambda)=\sum_{j=1}^{k} \lambda_{j}^{p} .
$$

Let $\alpha^{(j)}$ denote the first element of the Hurwitz tuple $h_{j}$, defined in (2). We define

$$
c_{p}^{*}(d, \Pi)=\sum_{j=1}^{\left|\operatorname{Cov}_{d}^{*}(\Pi)\right|} S_{p}\left(\alpha^{(j)}\right), \quad \text { and } \quad c_{p}^{*}(\Pi)=\sum_{d \geq 0} c_{p}^{*}(d, \Pi) q^{d},
$$

where for $* \in\left\{{ }^{\prime}, 0, \emptyset\right\}$ we packaged the Siegel-Veech weighted Hurwitz numbers into a generating series. These series admit the following graph sum decomposition.

Proposition 7.1. The generating series $c_{p}^{\prime}(\Pi)$ can be expressed in terms of graph sums of triple Hurwitz numbers as

$$
c_{p}^{\prime}(\Pi)=\sum_{\Gamma} \frac{1}{|\operatorname{Aut}(\Gamma)|} c_{p}^{\prime}(\Pi, \Gamma),
$$

where

$$
c_{p}^{\prime}(\Pi, \Gamma)=\sum_{G \in \Gamma} \sum_{\substack{h \in \mathbb{N}^{E}(G) \\ w \in \mathbb{Z}_{+}^{E(G)}}}\left(\sum_{e \in E(G)} h_{e} w_{e}^{p}\right) \prod_{e \in E(G)} w_{e} q^{h_{e} w_{e}} \prod_{v \in V(G)} A^{\prime}\left(\mathbf{w}_{v}^{-}, \mathbf{w}_{v}^{+}, \mu_{v}\right) \delta(v) .
$$

As a corollary of this and Theorem 7.3 below, we obtain an independent proof of the following quasimodularity result (see also CMZ16, Theorem 6.4]) without relying on the combinatorial machinery of $q$-brackets involving $\widetilde{T_{p}}$ (cf. CMZ16, Section 14 and 15]).

Corollary 7.2. For any ramification profile $\Pi$ and any odd $p \geq-1$ the generating series $c_{p}^{\prime}(\Pi)$ for counting covers without unramified components and with $p$-SiegelVeech weight as well as the generating series $c_{p}^{\circ}(\Pi)$ for connected counting with $p$-Siegel-Veech weight are quasi-modular forms of mixed weight $\leq \mathrm{wt}(\Pi)+p+1$.

7.1. Relation to area Siegel-Veech constants. The generating functions $c_{-1}^{\prime}(\Pi)$ admit a nice geometric interpretation in terms of Siegel-Veech constants, that are responsible for counting closed geodesics on flat surfaces. For a flat surface $X$ we define the counting function

$$
N_{\text {area }}(T, L)=\sum_{\substack{z \subset X \text { cylinder, } \\ w(Z) \geq L}} \frac{\operatorname{Area}(Z)}{\operatorname{Area}(X)} .
$$

counting the cylinders filled by closed geodesics on $X$, weighted by their area. This function is well known to have a quadratic asymptotic (see e.g. EM01), and the number

$$
c_{\text {area }}(X)=\lim _{L \rightarrow \infty} \frac{N_{\text {area }}(T, L)}{\pi L^{2}}
$$

is called the (area) Siegel-Veech constant associated to $X$. This constants are interesting both for generic flat surfaces of a given singularity type and for torus 
covers. It is shown in [EKZ14, Theorem 4] and [CMZ16, Theorem 3.1] that the Siegel-Veech constant for a torus cover of degree $d$ and ramification $\Pi$ is

$$
c_{\text {area }}(d, \Pi)=\frac{3}{\pi^{2}} \frac{c_{-1}^{0}(d, \Pi)}{N_{d}^{0}(\Pi)} .
$$

Thus the series $c_{-1}^{*}(\Pi)$ can be interpreted as generating functions for the "SiegelVeech numerators" of Hurwitz spaces. Knowing them to be quasimodular forms, and thus knowing the asymptotic behaviour of both $c_{-1}^{0}(d, \Pi)$ and $N_{d}^{0}(\Pi)$ as $d \rightarrow \infty$ allows to compute the area Siegel-Veech constant of a generic surface with a given singularity type.

7.2. Siegel-Veech weighted graph sums. In view of the Proposition 7.1 we consider now some variants of the graphs sums discussed Section [6] and show that they are quasimodular too. We define again the Siegel-Veech weighted graph sums

$$
S^{S V}(\Gamma, \mathbf{m})=\sum_{G \in \Gamma} S^{S V}(G, \mathbf{m})
$$

over all orientations $G$ of $\Gamma$, where now

$$
S^{S V}(G, \mathbf{m})=\sum_{h \in \widetilde{\mathbb{N}}^{E(G)}, w \in \mathbb{Z}_{+}^{E(G)}}\left(\sum_{i \in E(G)} \frac{h_{i}}{w_{i}}\right) \prod_{i \in E(G)} w_{i}^{m_{i}+1} q^{h_{i} w_{i}} \prod_{v \in V(G)} \delta(v)
$$

Theorem 7.3. If $\mathbf{m}=\left(m_{1}, \ldots, m_{|E(\Gamma)|}\right)$ is a tuple of even integers, then the graph sums $S^{S V}(\Gamma, \mathbf{m})$ are quasimodular forms of weight at most $k(\mathbf{m})=\sum_{i}\left(m_{i}+2\right)$.

As in the case of ordinary counting, we can split off the loops and reduce to a simplified height space. The main new ingredient is that nearly-elliptic function $L$ and $q$-derivatives of $P$ have the right Fourier expansion whose constant coefficients capture the new graph sums and still fit in the scope of quasimodularity results of Section 5.3 .

We decompose $S^{S V}(\Gamma, \mathbf{m})$ according to the edge $i_{0}$ that contributes $h_{i} / w_{i}$ in the prefactor. That is, we decompose

$$
S^{S V}(\Gamma, \mathbf{m})=\sum_{i_{0}} S_{i_{0}}^{S V}(\Gamma, \mathbf{m}), \quad \text { where } \quad S_{i_{0}}^{S V}(\Gamma, \mathbf{m})=\sum_{G \in \Gamma} S_{i_{0}}^{S V}(G, \mathbf{m})
$$

and where

$$
S_{i_{0}}^{S V}(G, \mathbf{m})=\sum_{h \in \widetilde{\mathbb{N}}^{E(G)}, w \in \mathbb{Z}_{+}^{E(G)}} \frac{h_{i_{0}}}{w_{i_{0}}} \prod_{i \in E(G)} w_{i}^{m_{i}+1} q^{h_{i} w_{i}} \prod_{v \in V(G)} \delta(v) .
$$

Next, we replace the height space $\widetilde{\mathbb{N}}^{E}(G)$ by $\mathbb{N}_{E(G)}$ using an analog of Lemma 6.3.

Lemma 7.4. Replacing the height space $\widetilde{\mathbb{N}}^{E(G)}$ by $\mathbb{N}_{E(G)}$ in each term $S^{S V}(G, \mathbf{m})$ does not change the total sum $S^{S V}(\Gamma, \mathbf{m})$.

Proof. Let $-G \in \Gamma$ be the graph with the reversed orientation compared to $G \in \Gamma$. We indicate by an additional index the space over which the summation $h$ is taken in $S^{S V}(G, \mathbf{m})$ We will show that

$$
S^{S V}(G, \mathbf{m})_{\widetilde{\mathbb{N}} E(G)}+S^{S V}(-G, \mathbf{m})_{\widetilde{\mathbb{N}} E(-G)}=S^{S V}(G, \mathbf{m})_{\mathbb{N}_{E(G)}}+S^{S V}(-G, \mathbf{m})_{\mathbb{N}_{E(-G)}} .
$$

On the first term of the left-hand side we apply the change of variables $h_{e}^{\prime}=h_{e}-\delta_{e}$ with $\delta_{e}=\varepsilon_{i^{+}(e)}-\varepsilon_{i^{-}(e)}$ for the orientation $G$, that maps $\widetilde{\mathbb{N}}^{E(G)}$ to $\mathbb{N}_{E(G)}$ as in 
Lemma 6.3. On the second term we apply the change of variables $h_{e}^{\prime}=h_{e}+\delta_{e}$ with $\delta_{e}$ as before, i.e. associated to the orientation $G$. It maps $\widetilde{\mathbb{N}}^{E(-G)}$ to $\mathbb{N}_{E(-G)}$. As in Lemma 6.3 this change of variable does not affect the term $q^{h \cdot w}$ (thanks to the delta functions). The statement is then obvious since the terms in $\delta_{e} / w_{e}$ cancel out.

We reduce the problem to the reduced graph using an analog of Lemma 6.4

Lemma 7.5. The Siegel-Veech weighted graph sums factor as

$$
S_{i_{0}}^{S V}(\Gamma, \mathbf{m})= \begin{cases}S_{0, i_{0}}^{S V}\left(\Gamma, \mathbf{m}_{0}\right) S\left(\Gamma_{1}, \mathbf{m}_{1}\right) & \text { if } i_{0} \text { is neutral, } \\ S_{0}\left(\Gamma, \mathbf{m}_{0}\right) S_{i_{0}}^{S V}\left(\Gamma_{1}, \mathbf{m}_{1}\right) & \text { otherwise, }\end{cases}
$$

where $\Gamma_{1}$ is the reduced graph underlying $\Gamma$ and where

$$
S_{0, i_{0}}^{S V}\left(\Gamma, \mathbf{m}_{0}\right)=\sum_{h \in \mathbb{N}_{E_{0}(G)}, w \in \mathbb{Z}_{+}^{E_{0}(G)}} \frac{h_{i_{0}}}{w_{i_{0}}} \prod_{i \in E_{0}(G)} w_{i}^{m_{i}+1} q^{h_{i} w_{i}}
$$

for any orientation $G$ of $\Gamma$.

The contribution of the loops is easily dealt with.

Lemma 7.6. For $\mathbf{m}$ a tuple of even integers $S_{0, i_{0}}^{S V}\left(\Gamma, \mathbf{m}_{0}\right)$ is a quasimodular form of mixed weight $k\left(\mathbf{m}_{0}\right)$.

Proof. Clearly

$$
S_{0, i_{0}}^{S V}\left(\Gamma, \mathbf{m}_{0}\right)=\tilde{S}_{m_{i_{0}}} \prod_{i \neq i_{0}} S_{m_{i}}
$$

where $S_{m}$ is defined in Lemma 6.5 and where

$$
\tilde{S}_{m_{i_{0}}}=\sum_{w, h=1}^{\infty} h w^{m_{i_{0}}} q^{h w}= \begin{cases}D_{q} S_{m_{i_{0}}}-2 & \text { if } m_{i_{0}} \geq 2 \\ E_{2}+\frac{1}{24} & \text { if } m_{i_{0}}=0 .\end{cases}
$$

Proof of Theorem 7.3. It remains to show that if $i_{0}$ is not neutral, then the graph sum $S_{i_{0}}^{S V}\left(\Gamma_{1}, \mathbf{m}_{1}\right)$ is a quasimodular form. We define

$$
P_{\Gamma_{1}, \mathbf{m}_{1}}^{S V, i_{0}}(\mathbf{z})=D_{q} P^{\left(m_{i_{0}}-2\right)}\left(z_{v_{1}\left(i_{0}\right)}-z_{v_{2}\left(i_{0}\right)}\right) \prod_{i \in E\left(\Gamma_{1}\right) \backslash\left\{i_{0}\right\}} P^{\left(m_{i}\right)}\left(z_{v_{1}(i)}-z_{v_{2}(i)}\right)
$$

if $m_{i_{0}} \geq 2$ and in the remaining case $m_{i_{0}}=0$ we let

$$
P_{\Gamma_{1}, \mathbf{m}_{1}}^{S V, i_{0}}(\mathbf{z})=L\left(z_{v_{1}\left(i_{0}\right)}-z_{v_{2}\left(i_{0}\right)}\right) \prod_{i \in E\left(\Gamma_{1}\right) \backslash\left\{i_{0}\right\}} P^{\left(m_{i}\right)}\left(z_{v_{1}(i)}-z_{v_{2}(i)}\right) .
$$

These definitions are designed such that, with the same proof as in Proposition 6.7 we obtain

$$
S_{i_{0}}^{S V}\left(\Gamma_{1}, \mathbf{m}_{1}\right)=\left[\zeta_{n}^{0} \ldots \zeta_{1}^{0}\right] P_{\Gamma_{1}, \mathbf{m}_{1}}^{S V, i_{0}}(\mathbf{z}) .
$$

By Proposition 5.6, the function $L$ belongs to $\mathcal{Q}_{n}^{(0)} \oplus \mathcal{Q}_{n}^{(2)}$, and the function $D_{q} P^{\left(m_{i_{0}}\right)}$ belongs to $\mathcal{Q}_{n}^{\left(m_{i_{0}}+2\right)}$. So in any case $P_{\Gamma_{1}, \mathbf{m}_{1}}^{S V, i_{0}}$ belongs to $\mathcal{Q}_{n}^{\left(k\left(\mathbf{m}_{1}\right)-2\right)} \oplus \mathcal{Q}_{n}^{\left(k\left(\mathbf{m}_{1}\right)\right)}$. Its constant term hence is quasimodular of mixed weight $\leq k\left(\mathbf{m}_{1}\right)$ by Theorem 5.8 . 


\subsection{Proof of main results.}

Proof of Proposition 7.1. As in the proof of Proposition 4.2 we rely on Proposition 2.4 We only need to justify that counting with Siegel-Veech weight produces an extra factor $h_{e} w_{e}^{p}$ for each edge. This is exactly the weight to put on each cylinder that corresponds to the weight $S_{p}(\lambda)$ for a Hurwitz tuple. This correspondence is obtained using standard Siegel-Veech transform as is the proof of Theorem 3.1 of [CMZ16].

Proof of Corollary 7.2. First, we claim that

$$
c_{p}^{\prime}(\Pi)=\left\langle T_{p} f_{\mu_{1}} \cdots f_{\mu_{n}}\right\rangle_{q}-\left\langle T_{p}\right\rangle_{q}\left\langle f_{\mu_{1}} \cdots f_{\mu_{n}}\right\rangle_{q}
$$

as difference of $q$-brackets, where $T_{p}$ is a function on partitions that we introduce now. With the definition 4

$$
T_{p}(\lambda)=\sum_{\tau \in \mathcal{P}(d)} z_{\tau} S_{p}(\tau) \chi^{\lambda}(\tau)^{2}
$$

Proposition 6.3 of [CMZ16] implies that

$$
c_{p}(\Pi)=\sum_{\lambda \in \mathbb{P}}\left(T_{p} f_{\mu_{1}} \cdots f_{\mu_{n}}\right)(\lambda) q^{\lambda} .
$$

From [CMZ16, Proposition 6.2 we deduce

$$
c_{p}^{\prime}(\Pi)=(q)_{\infty} c_{p}(\Pi)-(q)_{\infty} c_{p}() N_{p}^{\prime}(\Pi) .
$$

Since $(q)_{\infty}=\left(\sum_{\lambda \in \mathbb{P}} q^{\lambda}\right)^{-1}$, this is equivalent to the equation claimed in (59), by the definition of $q$-brackets.

Second, we use the linearity of the brackets to show

$$
\begin{aligned}
& \left\langle T_{p} p_{\ell_{1}} \cdots p_{\ell_{n}}\right\rangle_{q}-\left\langle T_{p}\right\rangle_{q}\left\langle p_{\ell_{1}} \cdots p_{\ell_{n}}\right\rangle_{q}
\end{aligned}
$$

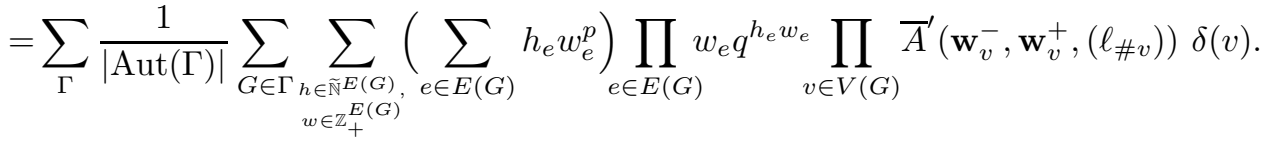

which invokes the polynomial Hurwitz numbers $\bar{A}^{\prime}\left(\mathbf{w}_{v}^{-}, \mathbf{w}_{v}^{+},\left(\ell_{\# v}\right)\right)$. In fact, we can consider both sides of this equation as expressions in $n$ arguments as in Theorem 4.3 . Since we can write $p_{\ell}=\sum_{\mu} c_{\ell, \mu} f_{\mu}$, multilinearity of both sides reduces the claim to the case of arguments $f_{\mu_{i}}$, which is exactly the combination of (59) and the claim in Proposition 7.1

By Theorem 4.1 the polynomials $\bar{A}^{\prime}\left(\mathbf{w}_{v}^{-}, \mathbf{w}_{v}^{+},\left(\ell_{\# v}\right)\right)$ are even, so if $p$ is odd the parity hypothesis of Theorem 7.3 is met and this theorem implies that the expression in (60) is a quasimodular form. Using that the $f_{\mu}$ can be expressed as polynomials

${ }^{4}$ This function arise more naturally as the hook length moment

$$
T_{p}(\lambda)=\sum_{m=1}^{\infty} m^{p-1} N_{m}(\lambda),
$$

where $N_{m}(\lambda)$ is the number of cells in the Young diagram of $\lambda$ of hook length $m$. That $T_{p}$ is a shifted symmetric function if and only if $p$ is odd and positive was a main theme in [CMZ16], but all this is not relevant here. That this definition coincides with the definition of $T_{p}$ given here is proven in [CMZ16], Corollary 13.2. 
in the $p_{\ell}$ by Theorem 3.1, this implies the quasimodularity of $c_{p}^{\prime}(\Pi)$ we claimed. The weight can be determined as in the proof of Corollary 6.2.

\section{TROPICAL COVERS AND QUASIMODULARITY GRAPH BY GRAPH}

The main result of BBBM13 is the expression of the tropical Hurwitz number generating function in term of a sum over Feynman graphs. The goal of this section is to show that their results are the special case of simple branch points of our results, when stated in the language of tropical covers. More precisely, we show here that our correspondence theorem Proposition 2.4 has the Correspondence Theorem BBBM13, Theorem 2.13] (see also Theorem 2.20 and Theorem 2.30 in loc. cit.) as immediate corollary when stated in the language of tropical covers. In particular, for simple branching (i.e. ramification profile $\Pi=((2), \ldots,(2))$, our counting problem is equivalent to counting tropical covers.

8.1. Tropical covers. We recall here the definition of a tropical curve and a tropical cover, following [BBBM13].

Definition 8.1. A tropical curve $C$ is a connected finite trivalent metric graph. An elliptic tropical curve consists of one edge forming a circle of certain length. Let $E$ be the elliptic tropical curve of length 1. A map $\pi: C \rightarrow E$ is a tropical cover of $E$, if it is continuous, non-constant, integer affine on each edge and respects a balancing condition at every vertex of $C$.

In this setting, the weight $w_{e}$ of an edge $e$ for the graph is defined as the slope of $\pi_{\mid e}$, and the degree of the cover as

$$
d=\sum_{P \in C, \pi(P)=p} w_{e_{P}}
$$

where $p$ is a generic point. For each vertex we can group the outgoing half-edges according to the half edges of $E$ they map to. The cover is called balanced if for each vertex the sum of the weights of the two groups agree.

The appropriate way to count tropical covers is coded in the notion of tropical Hurwitz numbers.

Definition 8.2. Fix branch points $p_{1}, \ldots p_{2 g-2}$ in the tropical elliptic curve $E$. The tropical Hurwitz number is the weighted number of isomorphism classes of degreed covers from a genus $g$ curve $C$, having the branch points at the $p_{i}$. Here, a tropical cover $\pi$ is weighted by the multiplicity

$$
\operatorname{mult}(\pi)=\frac{1}{|\operatorname{Aut}(\pi)|} \prod_{e} w_{e} .
$$

The combinatorial type of a tropical curve is its homeomorphism class, i.e. the underlying graph without length on the edges. These graphs are called Feynman graphs in physics literature. They correspond to our notion of (associated) global graph, with the vertex labeling removed.

Our correspondence theorem implies the following correspondence result for simple ramification covers branched over $n=2 g-2$ points in term of tropical covers. The number of covers is independent, both for flat surface covers and for tropical covers, on the base elliptic curve and the branch point location. In the following corollary we thus fix $E$ and the branch points on the flat side as in Section 2.3. 
more precisely for convenience to be $\varepsilon_{i}=i / n$ and on the tropical side we locate the branch points at $p_{i}=i / n$. In particular the heights $h_{e} \in \frac{1}{n} \mathbb{Z}_{\geq 1}$.

Corollary 8.3. There is a bijective correspondence between

i) flat surfaces $(X, \omega)$ with connected coverings $p: X \rightarrow E$ of degree $d$ of the square torus $E$, with $\omega=p^{*} \omega_{E}$, and with simple ramification profile, weighted by $1 /|\operatorname{Aut}(\pi)|$, and

ii) isomorphism classes of weighted tropical covers $\pi: C \rightarrow E$ of degree $d$, where the weight of a tropical cover corresponds to its multiplicity $\operatorname{mult}(\pi)$.

Proof. This is a consequence of Proposition 2.4. Each cover corresponds to a trivalent graph with a collection of numbers $\left(w_{e}, h_{e}, t_{e}\right)$ corresponding to the widths, the heights and the twists of the cylinders. We define the tropical curve $C$ to be the global graph $\Gamma$ with edge lengths $\ell_{e}=h_{e} / w_{e}$. If $e$ is an edge from vertex $i$ to vertex $j$ we define the tropical cover on $e$ to be the map of slope $w_{e} \in \mathbb{N}$ to the multi-segment from $p_{i}$ to $p_{j}$ making $\left\lfloor h_{e}\right\rfloor$ full turns. Note that this is well-defined, since for such an edge $h_{e}-(j-i) / n \in \mathbb{Z}$. The tropical balancing condition is a restatement of $\left|\mathbf{w}_{v}^{+}\right|=\left|\mathbf{w}_{v}^{-}\right|$for every vertex $v$. Note that on the one hand $d=\sum_{e} w_{e} h_{e}$, but on the other hand the flat picture immediately implies $d=\sum_{e \ni \pi^{-1}(P)} w_{e}$, where the sum is over all edges $e$ such that the corresponding cylinder contains the preimage of a given point $P \in E$. This implies that the tropical cover we defined has indeed degree $d$. The map we define forgets the twist $t_{e}$, but this is accounted for in the multiplicity mult $(\pi)$. Note that the double Hurwitz number of a trivalent local surface with simple branch point is equal to one and can hence be omitted. Besides the twist, our map has an obvious converse, associating to a tropical cover the slopes $w_{e}$ and $h_{e}=w_{e} \ell_{e}$ and the latter are indeed in $\frac{1}{n} \mathbb{Z}_{\geq 1}$ by our convention on the location of the points $p_{i}$.

Proposition 2.4 is stated at the level of coverings without unramified components. The correspondence descends under the given hypothesis of simple branching (more generally: in case of only one ramified point over each branch point) to a correspondence of connected covers by the usual inclusion-exclusion principle, since the obstruction of disconnected local surfaces (mentioned after the proof of Proposition 2.4) is ruled out by this hypothesis.

In this correspondence the lengths of the edges of the tropical curves are the reciprocal of the modulus $m_{e}=w_{e} / h_{e}$. This is the natural choice viewing tropical curves as the dual graph of the special fiber in a degenerating family of smooth curves. Indeed the reciprocal moduli $m_{e}^{-1}$ of the cylinders correspond (up to a common rescaling) to the number of Dehn twists performed under the mondromy around the special fiber and this in turn corresponds to a local equation $x y=t^{m_{e}^{-1}}$ in the stable model of the generating fiber (see e.g. Möl08, paragraph preceding Theorem 2.4). Since such a singularity is resolved by a chain of $m_{e}^{-1}-1$ rational curves in the semistable model with regular total space, the tropicalization map (Viv13]) provides this edge with length $m_{e}^{-1}$.

8.2. Counting graph by graph. For coverings with simple ramification, or equivalently for trivalent graphs, the quasimodularity results hold for each individual graph. 
Corollary 8.4. Let $\Pi=((2), \ldots,(2))$. Then for any trivalent graph $\Gamma$ the contribution $N^{\prime}(\Pi, \Gamma)$ (resp $c_{p}^{\prime}(\Pi, \Gamma)$ ) of the graph $\Gamma$ to the total counting is a quasimodular form of mixed weight less or equal to $\mathrm{wt}(\Pi)=|\Pi|+\ell(\Pi)$.

These graph sums have a geometric interpretation: we count only surfaces with a fixed type. This result is a refinement of the quasimodularity results of EO01. and CMZ16] in the case of the principal strata.

Note that the weight of these quasimodular form $N^{\prime}(\Pi, \Gamma)$ is not necessary pure as shown by the example of $\Pi=((2),(2),(2),(2))$ in Section 9 . Note that in our convention, the vertices of $\Gamma$ are labeled. Our examples show that for fixed underlying unlabeled trivalent graph $G$ the sum over all labelings is a quasimodular form of pure weight $\mathrm{wt}(\Pi)=|\Pi|+\ell(\Pi)$ for $\Pi=((2),(2))$ and $\Pi=((2),(2),(2),(2))$. This purity result might hold in general.

Proof. Since for simple ramification $f_{2}=P_{2} / 2$ is a completed cycle, the corollary is a straightforward consequence of Theorem 6.1 and Theorem 7.3 .

\section{EXAMPLES}

The examples here have four objectives. First we show how to compare the computations of Zorich in Zor02 diagram by diagram with the counting by global graphs and local surfaces used here. Second, we emphasize the main difficulty in the naive computation of the graph sums: Working with non-completed cycles the graph sum is not quasimodular for each graph separately, non even after summing over all the orientations. This sum belongs to the ring generated by all the "Eisenstein series" $G_{k}$, including odd $k$ (compare section [5.1), and its derivatives. Only cancellations that become very delicate as the complexity of the ramification datum grows ensure that the total result is a quasimodular form.

Third, we illustrate the mechanism for proving quasimodularity of the SiegelVeech weighted counting. This is most transparent in the case of the principal stratum in genus two, the simple branching profile $\Pi=((2),(2))$, where no difficulty stemming from completed cycles is present.

Finally, we compute the quasimodular forms individually for the trivalent graphs corresponding to genus three covers and $\Pi=((2),(2),(2),(2))$.

9.1. Branching profile $\Pi=(3)$, the stratum $\mathcal{H}(2)$. Counting geometrically as in Section 2.3, the global graphs of a stratum with just one singularity have just one node, and the number of loops is at most two loops for genus two curves.
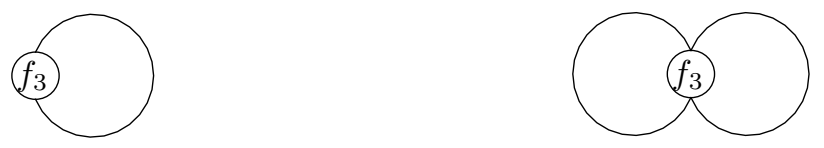

Figure 3 . The global graphs for $\mathcal{H}(2)$ : One loop $\left(\Gamma_{1}\right)$ or two loops $\left(\Gamma_{2}\right)$. 
9.1.1. Computation by diagrams. We review the computations of Zorich in [Zor02] of torus covers in this stratum, made with the aim of computing the Masur-Veech volume of $\mathcal{H}(2)$. We compute $N^{\circ}(G, \Pi)=N^{\prime}(G, \Pi)$ for $\Gamma_{1}$ and $\Gamma_{2}$ and all their orientations for the ramification profile $\Pi=(3)$ consisting of a three-cycle.

In terms of square-tiled surfaces in $\mathcal{H}(2)$, a first possible pattern is presented on the left picture of Figure 4. The picture on the right represents the ribbon graph made from a tubular neighborhood of the boundary of the horizontal cylinder. It is drawn on a torus since it can not be embedded in the plane. It corresponds to the only one ribbon graph with one vertex of valency 6 and 2 faces (boundary components).
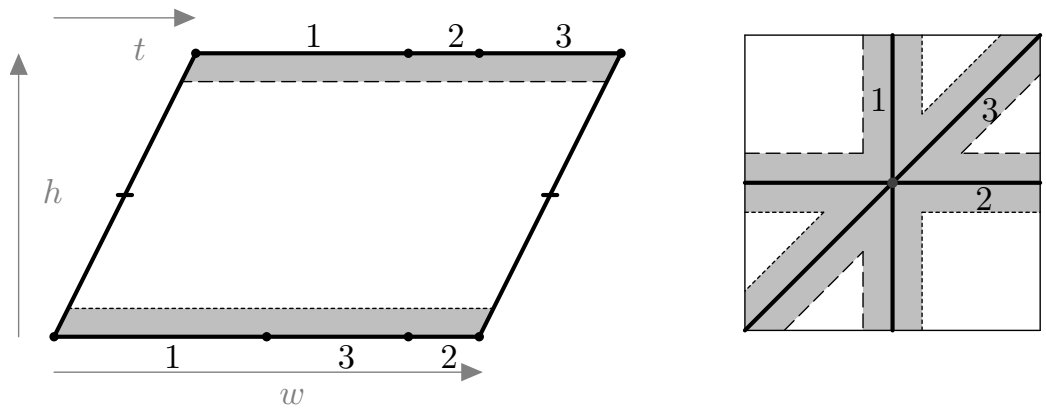

FIgURE 4. Diagram with one cylinder

As integer parameters for this square-tiled surface, we use the width of the cylinder $w$, the height $h$, the twist $t$, and the lengths of the saddle connexions $\ell_{1}, \ell_{2}, \ell_{3}$. They are related by

$$
w=\ell_{1}+\ell_{2}+\ell_{3}, \text { and } t \in\{0,1, \ldots, w-1\} .
$$

The generating function for square-tiled surfaces of this type is then

$$
\begin{aligned}
S\left(\mathcal{C}_{1}\right) & :=\sum_{\substack{h, \ell_{1}, \ell_{2}, \ell_{3} \\
h w \geq 3}}^{\infty} \sum_{t=0}^{w-1} q^{h w} \delta\left(w-\ell_{1}-\ell_{2}-\ell_{3}\right) \\
& =\sum_{\substack{w, h=1 \\
h w \geq 3}}^{\infty} w q^{h w}\left(\frac{1}{6} w^{2}-\frac{1}{2} w+\frac{1}{3}\right)=\frac{1}{6} S_{3}-\frac{1}{2} S_{2}+\frac{1}{3} S_{1},
\end{aligned}
$$

where $\frac{1}{6} w^{2}-\frac{1}{2} w+\frac{1}{3}$ is the number of solutions of (61), and where

$$
S_{i}=\sum_{w, h=1}^{\infty} w^{i} q^{h w}=G_{i+1}+\frac{B_{i+1}}{2(i+1)} .
$$

From this formula we see that this generating function $N^{\prime}\left(\Gamma_{1},(3)\right)$ is not a quasimodular form of weight $\leq 6$, since the "Eisenstein series" $G_{3}$ isn't.

Figure 5 represents a pattern for a square-tiled surface in $H(2)$ corresponding to the graph $\Gamma_{2}$, i.e., with 2 horizontal cylinders, and its associated ribbon graph. The integer parameters $w_{1}, w_{2}, h_{1}, h_{2}, t_{1}, t_{2}, \ell_{1}, \ell_{2}, \ell_{3}$ are related by 

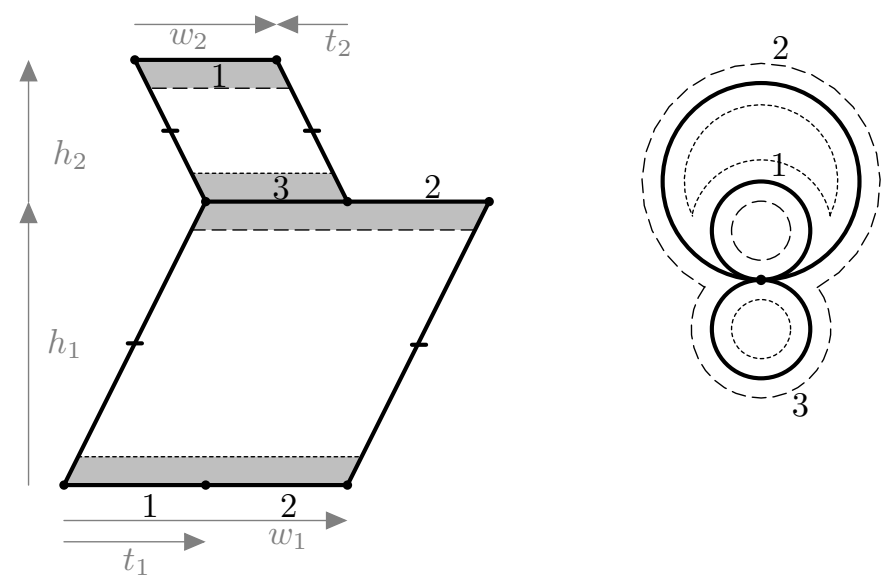

FiguRE 5. Diagram for two cylinders

$$
w_{2}=\ell_{1}=\ell_{3}, w_{1}=\ell_{1}+\ell_{2}, t_{1} \in\left\{0, \ldots, w_{1}-1\right\}, t_{2} \in\left\{0, \ldots, w_{2}-1\right\} .
$$

The generating function for this type of square-tiled surfaces is

$$
\begin{aligned}
S\left(\mathcal{C}_{2}\right) & :=\sum_{\substack{w_{1}, h_{1}, w_{2}, h_{2}, \ell_{1}, \ell_{2}=1 \\
w . h \geq 3}}^{\infty} \sum_{t_{1}=0}^{w_{1}-1} \sum_{t_{2}=0}^{w_{2}-1} q^{h_{1} w_{1}+h_{2} w_{2}} \delta\left(w_{2}-\ell_{1}\right) \delta\left(w_{1}-\ell_{1}-\ell_{2}\right) \\
& =\sum_{\substack{w_{1}, w_{2}, h_{1}, h_{2}=1 \\
w . h \geq 3}}^{\infty} w_{1} w_{2} q^{h_{1} w_{1}+h_{2} w_{2}} \mathbb{1}_{\left\{w_{1}>w_{2}\right\}} .
\end{aligned}
$$

We now compute that

$$
\sum_{w_{1}, w_{2}, h_{1}, h_{2}=1}^{\infty} w_{1} w_{2} q^{h_{1} w_{1}+h_{2} w_{2}} \mathbb{1}_{\left\{w_{1}>w_{2}\right\}}=\frac{1}{2}(A-B),
$$

where

$$
A=\sum_{w_{1}, w_{2}, h_{1}, h_{2}=1}^{\infty} w_{1} w_{2} q^{h_{1} w_{1}+h_{2} w_{2}}=S_{1}^{2}
$$

and

$$
B=\sum_{w_{1}, w_{2}, h_{1}, h_{2}=1}^{\infty} w_{1} w_{2} q^{h_{1} w_{1}+h_{2} w_{2}} \mathbb{1}_{\left\{w_{1}=w_{2}\right\}}=D_{q} S_{1}-S_{2}
$$

Here again, the non-quasimodularity comes from the factor $S_{2}=G_{3}$. Summing over the two configurations we see that the term in $S_{2}$ cancel out,

$$
\left(\frac{1}{6} S_{3}-\frac{1}{2} S_{2}+\frac{1}{3} S_{1}\right)+\frac{1}{2}\left(S_{1}^{2}-D S_{1}+S_{2}\right)=\frac{3}{2} G_{2}^{2}-\frac{1}{4} G_{4}+\frac{3}{8} G_{2}+c s t
$$

resulting in a quasimodular form, as claimed.

9.1.2. Computation by local graphs and global graphs. In the formalism of this paper, the ribbon graph in the first case (i.e. the global graph $\Gamma_{1}$ ) corresponds to a cover of the cylinder $\mathbb{P}^{1}$, ramified with profile $(3,1, \ldots, 1)$ at 1 , and with $(w)$ at 0 and $\infty$. The corresponding triple Hurwitz number $A^{\prime}(w, w,(3))$ is just the number 
of such ribbon graphs, so it is the number of solutions $\left(\ell_{1}, \ell_{2}, \ell_{3}\right) \in \mathbb{N}^{3}$ of (61). Consequently,

$$
S\left(\mathcal{C}_{1}\right)=N^{\prime}\left(\Gamma_{1},(3)\right)=\sum_{w, h=1}^{\infty} w q^{w h} A^{\prime}(w, w,(3))
$$

For the second graph $\Gamma_{2}$, the ribbon graph corresponds here to a cover of $\mathbb{P}^{1}$, ramified of profile $(3,1, \ldots, 1)$ over $1,\left(w_{1}, w_{2}\right)$ over 0 and $\infty$. The number of such covers or such ribbon graphs is

$$
A^{\prime}(\mathbf{w}, \mathbf{w},(3))=\left\{\begin{array}{l}
1 \text { if } w_{1} \neq w_{2} \\
0 \text { if } w_{1}=w_{2},
\end{array} \quad\left(\mathbf{w}=\left(w_{1}, w_{2}\right)\right) .\right.
$$

Before passing to the computation using completed cycles, we tabulate the contribution of each polynomial in shifted symmetric functions appearing in the expression of

$$
f_{3}=\frac{1}{3} P_{3}-\frac{1}{2}\left(P_{1}\right)^{2}+\frac{5}{12} P_{1}
$$

to the local polynomials $A^{\circ}\left(\mathbf{w}^{-}, \mathbf{w}^{+},(3)\right)$ and the contribution $\langle\cdot\rangle_{q, G}$. The table below shows that the contribution of each diagram individually is not quasimodular because the contribution of $P_{1}^{2}$ for graphs with only one vertex is not polynomial. It is however piecewise polynomial, showing also that all the hypothesis in Theorem 4.1 are needed in order to get a globally polynomial contribution.

\begin{tabular}{|c|c|c|c|c|c|}
\hline & $A^{\circ}(w, w,(3))$ & $\langle\cdot\rangle_{q, G_{1}}$ & \multicolumn{2}{|c|}{$A^{\circ}(\mathbf{w}, \mathbf{w},(3))$} & $\langle\cdot\rangle_{q, G_{2}}$ \\
\hline & & & $w_{1} \neq w_{2}$ & $w_{1}=w_{2}$ & \\
\hline$f_{3}$ & $\frac{1}{6} w^{2}-\frac{1}{2} w+\frac{1}{3}$ & $\frac{1}{6} S_{3}-\frac{1}{2} S_{2}+\frac{1}{3} S_{1}$ & 1 & 0 & $S_{1}^{2}-D S_{1}+S_{2}$ \\
\hline$\frac{P_{3}}{3}$ & $\frac{1}{6} w^{2}-\frac{1}{12}$ & $\frac{1}{6} S_{3}-\frac{1}{12} S_{1}$ & 2 & 2 & $2 S_{1}^{2}$ \\
\hline$P_{1}^{2}$ & $w$ & $S_{2}$ & 2 & 4 & $2\left(S_{1}^{2}+D S_{1}-S_{2}\right)$ \\
\hline$P_{1}$ & 1 & $S_{1}$ & 0 & 0 & 0 \\
\hline
\end{tabular}

9.1.3. Computation using q-brackets of completed cycles. Last, we compute $\left\langle f_{3}\right\rangle_{q}$ using

$$
\left\langle f_{3}\right\rangle_{q}=\frac{1}{3}\left\langle P_{3}\right\rangle_{q}-\frac{1}{2}\left\langle P_{1}^{2}\right\rangle_{q}+\frac{5}{12}\left\langle P_{1}\right\rangle_{q}
$$

The main difference with the previous computation will be the term $\left\langle P_{1}^{2}\right\rangle_{q}$ that we could interpret as a graph sum for a graph with two vertices. 


\begin{tabular}{|c|c|c|}
\hline & $A^{\circ}\left(\mathbf{w}^{-}, \mathbf{w}^{+}, \mu\right)$ & $\langle\cdot\rangle_{q, G}$ \\
\hline & & 6 \\
\hline
\end{tabular}

In these cases, all the local contribution are globally polynomials and moreover even functions, so the contribution of the completed cycles for each graph are quasimodular forms.

9.2. Branching profile $\Pi=((2),(2))$, the stratum $\mathcal{H}(1,1)$. For this stratum, as for all principal strata, all the contributions of the individual graphs to the counting function $N^{\circ}((12),(12))$ for the ramification profile consisting of two transpositions are quasimodular forms, since $f_{2}=\frac{1}{2} P_{2}$ is equal to a completed cycle. For $\mathcal{H}(1,1)$ there is only one possible global graph up to relabeling the vertices.

\begin{tabular}{|c|c|c|}
\hline & $A^{\circ}\left(\left(w_{1}\right),\left(w_{2}, w_{3}\right),(2)\right)$ & $\left\langle P_{2}\right\rangle_{q, G}$ \\
\hline$P_{2}$ & & \\
\hline & 2 and 2 & $2 S_{1,1}$ \\
\hline
\end{tabular}

Among the 8 orientations of $\Gamma$ the two with all arrows ending at the same vertex do not contribute to the total sum (27). By symmetry considerations the remaing 6 orientations have the same contribution, and contribute with the factor $1 / 6$ to (27). because of the automorphism group permuting the edges. Consequently,

$$
N^{\circ}((12),(12))=\frac{1}{6} S(\Gamma), \quad \text { where } S(\Gamma)=\left[\zeta_{1}^{0} \zeta_{2}^{0}\right] P^{3}\left(z_{1}-z_{2}\right)=\left[\zeta^{0}\right] P^{3}(z)
$$

and on the other hand (expressed in the height space convention of Lemma 6.3)

$$
S(\Gamma)=6 S_{1,1} \quad \text { where } \quad S_{1,1}=\sum_{w_{2}, w_{3}, h_{1}=1, h_{2}, h_{3}=0}^{\infty} w_{2} w_{3}\left(w_{2}+w_{3}\right) q^{w_{2}\left(h_{1}+h_{2}\right)+w_{3}\left(h_{1}+h_{3}\right)}
$$


We can compute the constant coefficient using the algorithm provided by Theorem 5.8 and the decomposition

$$
P(z)^{3}=\frac{1}{120} P^{(4)}(z)+G_{2} P^{\prime \prime}(z)+\left(12 G_{2}^{2}+3 G_{4}\right) P(z)-16 G_{2}^{3}+4 G_{4} G_{2}+\frac{7}{30} G_{6} .
$$

to obtain finally that

$$
N^{\circ}((12),(12))=-\frac{8}{3} G_{2}^{3}+\frac{2}{3} G_{4} G_{2}+\frac{7}{180} G_{6} .
$$

As a cross-check, since we know that the graph sum is quasimodular, we can determine this quasimodular form, by computing the first terms of the generating series

$$
N^{\circ}((12),(12))=2 q^{2}+16 q^{3}+60 q^{4}+160 q^{5}+360 q^{6}+672 q^{7}+1240 q^{8}+O\left(q^{9}\right)
$$

9.2.1. Siegel-Veech generating function. Here we illustrate the method of proof of Theorem 7.3 by evaluating the first interesting contribution $S^{S V}(\mathcal{H}(1,1))$. We use the branch point normalization of heights 0 and $1 / 2$ (to exploit the symmetry). The generating function is

$$
S_{1,1}^{S V}=\sum_{\substack{\left(h_{1}, h_{2}, h_{3}\right) \in(\mathbb{N}+1 / 2)^{3} \\\left(w_{1}, w_{2}, w_{3}\right) \in\left(\mathbb{N}^{*}\right)^{3}}}\left(\frac{h_{1}}{w_{1}}+\frac{h_{2}}{w_{2}}+\frac{h_{3}}{w_{3}}\right) w_{1} w_{2} w_{3} \delta\left(w_{3}-w_{1}-w_{2}\right) q^{h . w} .
$$

We will show that

$$
S_{1,1}^{S V}=-\frac{10}{3} G_{2}^{3}+\frac{5}{6} G_{2} G_{4}+\frac{7}{144} G_{6} .
$$

With our convention, since the polynomial contribution of $P_{2}$ is 1 , we get

$$
c^{\circ}((12),(12))=\frac{1}{6}\left(S_{1}^{S V}(\Gamma, 0)+S_{2}^{S V}(\Gamma, 0)+S_{3}^{S V}(\Gamma, 0)\right)
$$

where

$$
S_{1}^{S V}(\Gamma, 0)=\sum_{G \in \Gamma} \sum_{\substack{w \in \mathbb{N}^{*} \\ h \in \mathbb{Z}_{E(G)}}} h_{1} w_{2} w_{3} \delta\left(\sum_{i \in e^{+}(v)} w_{i}-\sum_{i \in e^{-}(v)} w_{i}\right) q^{h \cdot w} .
$$

One can check directly on this example that

$$
S_{1}^{S V}(\Gamma, 0)=\left[\zeta^{0}\right] L(z) P^{2}(z)=S_{2}^{S V}(\Gamma, 0)=S_{3}^{S V}(\Gamma, 0)
$$

(the integration with respect to the second variable $z_{2}$ is not necessary), and that

$$
S_{1,1}^{S V}=\frac{1}{2} S_{1}^{S V}(\Gamma, 0)=c^{\circ}((12),(12)) .
$$

The proof of Theorem 7.3 also provides an algorithm for computation. The decomposition of $L$ in the standard generators has been given in (46). Using

$$
P^{2}(z)=\frac{1}{6} P^{\prime \prime}(z)+4 G_{2} P(z)-4 G_{2}^{2}+\frac{5}{3} G_{4}
$$

and the decomposition of $P^{3}(z)$ given previously, we obtain

and

$$
\left[\zeta^{0}\right] Z^{2}(z) P^{2}(z)=\frac{16}{3} G_{2}^{3}-\frac{2}{3} G_{2}^{2}-\frac{8}{3} G_{4} G_{2}+\frac{5}{18} G_{4}+\frac{7}{180} G_{6}
$$

$$
\left[\zeta^{0}\right]\left(\frac{1}{2} P(z)-G_{2}+\frac{1}{12}\right) P^{2}(z)=-4 G_{2}^{3}-\frac{1}{3} G_{2}^{2}+\frac{1}{3} G_{4} G_{2}+\frac{5}{36} G_{4}+\frac{7}{60} G_{6} .
$$


Taken together, we conclude that

$$
c^{\circ}((12),(12))=\frac{1}{2}\left[\zeta^{0}\right] L P^{2}=-\frac{10}{3} G_{2}^{3}+\frac{5}{6} G_{2} G_{4}+\frac{7}{144} G_{6}=\frac{5}{4} S(\mathcal{H}(1,1)) .
$$

The Siegel-Veech constant for the stratum $\mathcal{H}(1,1)$ is $5 / 4$ and this proportionality of generating series is expected by the "non-varying property" of $\mathcal{H}(1,1)$, see the discussion in CMZ16, Section 17].

9.3. Branching profile $\Pi=((2),(2),(2),(2))$, the stratum $\mathcal{H}\left(1^{4}\right)$. We end this range of examples with the stratum $\mathcal{H}\left(1^{4}\right)$, first to show the effect of the labeling of the zeros and to compute the quasimodular forms for individual graphs. There are only two trivalent connected (multi)graphs with 4 vertices, depicted in Figure 6. The graphs $\Gamma$ are obtained by labeling the vertices of these graphs.
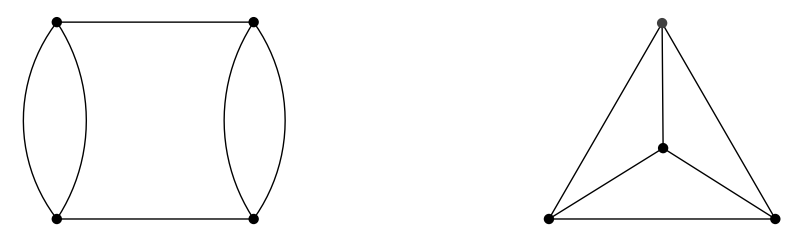

Figure 6. Global graphs for $\mathcal{H}\left(1^{4}\right)$ : Type 1 (left) and type 2 (right)

For the first graph, we can use the horizontal and vertical flip and assume that the bottom left vertex is labeled by one. Among the 6 ways to label the remaining vertices, our normalization of the integration paths (or equivalently height spaces, see 5.3), results in two essentially different quasimodular forms.
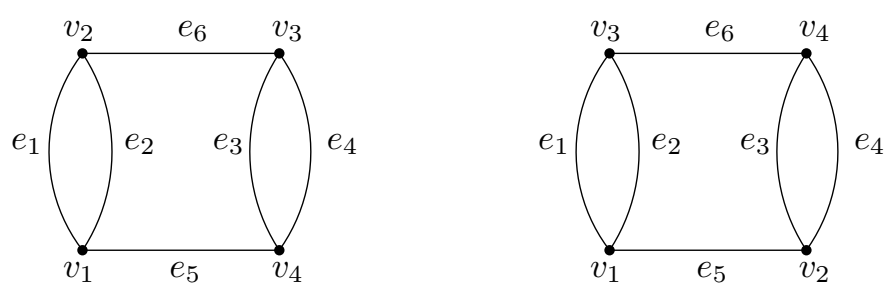

FiguRE 7. Labeled graph of type 1 for $\mathcal{H}\left(1^{4}\right)$

The numbering on the left gives the quasimodular form

$$
\begin{aligned}
A= & {\left[\zeta^{0}\right] P\left(z_{1}-z_{2}\right)^{2} P\left(z_{1}-z_{4}\right) P\left(z_{2}-z_{3}\right) P\left(z_{3}-z_{4}\right)^{2} } \\
= & 4 q^{2}+224 q^{3}+3088 q^{4}+21888 q^{5}+105136 q^{6}+388288 q^{7}+1197280 q^{8}+O\left(q^{9}\right) \\
= & \left(-256 G_{2}^{6}+\frac{640}{3} G_{4} G_{2}^{4}+\frac{112}{9} G_{6} G_{2}^{3}-\frac{400}{9} G_{4}^{2} G_{2}^{2}-\frac{140}{9} G_{6} G_{4} G_{2}+\frac{2000}{81} G_{4}^{3}\right. \\
& \left.+\frac{49}{108} G_{6}^{2}\right)+\left(-\frac{256}{3} G_{4} G_{2}^{3}-\frac{16}{5} G_{6} G_{2}^{2}+\frac{320}{21} G_{4}^{2} G_{2}+\frac{28}{9} G_{6} G_{4}\right)
\end{aligned}
$$


that we computed in Section 5.4 and there is a second labeling that results in the same quasimodular form, in fact

$$
A=\left[\zeta^{0}\right] P\left(z_{1}-z_{4}\right)^{2} P\left(z_{1}-z_{2}\right) P\left(z_{2}-z_{3}\right)^{2} P\left(z_{3}-z_{4}\right) .
$$

The numbering on the right of Figure 7 and three other numberings produce the quasimodular form

$$
\begin{aligned}
B= & {\left[\zeta^{0}\right] P\left(z_{1}-z_{3}\right)^{2} P\left(z_{1}-z_{2}\right) P\left(z_{2}-z_{4}\right)^{2} P\left(z_{3}-z_{4}\right) } \\
= & 40 q^{4}+448 q^{5}+2848 q^{6}+11776 q^{7}+41744 q^{8}+O\left(q^{9}\right) \\
= & \left(-256 G_{2}^{6}+\frac{640}{3} G_{4} G_{2}^{4}+\frac{112}{9} G_{6} G_{2}^{3}-\frac{400}{9} G_{4}^{2} G_{2}^{2}-\frac{140}{9} G_{6} G_{4} G_{2}+\frac{2000}{81} G_{4}^{3}\right. \\
& \left.+\frac{49}{108} G_{6}^{2}\right)+\left(\frac{128}{3} G_{4} G_{2}^{3}+\frac{8}{5} G_{6} G_{2}^{2}-\frac{160}{21} G_{4}^{2} G_{2}-\frac{14}{9} G_{6} G_{4}\right) .
\end{aligned}
$$

This difference illustrates a wall-crossing phenomenon: fixing the heights of the zeros (the domain of integration) and changing the labeling of the zeros is the same as fixing the labeling of the zeros and changing the integration domain. Equations (64) and (65) are the contour integral of the same function on two different domains. Note also that these contributions are of weight 12 and 10, and their weight 12 part coincide, whereas their weight 10 part coincide up to a factor -2 .

The total contribution of this graph is then

$$
\begin{aligned}
2 A+4 B=6 & \left(-256 G_{2}^{6}+\frac{640}{3} G_{4} G_{2}^{4}+\frac{112}{9} G_{6} G_{2}^{3}-\frac{400}{9} G_{4}^{2} G_{2}^{2}-\frac{140}{9} G_{6} G_{4} G_{2}\right. \\
& \left.+\frac{2000}{81} G_{4}^{3}+\frac{49}{108} G_{6}^{2}\right) .
\end{aligned}
$$

The second graph on Figure 6 is totally symmetric: there is only one way to label the vertices. Its contribution is

$$
\begin{aligned}
C & =\left[\zeta^{0}\right] P\left(z_{1}-z_{2}\right) P\left(z_{1}-z_{3}\right) P\left(z_{1}-z_{4}\right) P\left(z_{2}-z_{3}\right) P\left(z_{2}-z_{4}\right) P\left(z_{3}-z_{4}\right) \\
& =-384 G_{2}^{6}+480 G_{4} G_{2}^{4}-200 G_{4}^{2} G_{2}^{2}+\frac{250}{9} G_{4}^{3}
\end{aligned}
$$

The total connected generating function for the graph of type 1 is then

$$
\begin{aligned}
& \frac{1}{4}(2 A+4 B)+C \\
= & -768 G_{2}^{6}+800 G_{4} G_{2}^{4}+\frac{56}{3} G_{6} G_{2}^{3}-\frac{800}{3} G_{4}^{2} G_{2}^{2}-\frac{70}{3} G_{6} G_{4} G_{2}+\frac{1750}{27} G_{4}^{3}+\frac{49}{72} G_{6}^{2} \\
= & 2 q^{2}+160 q^{3}+2448 q^{4}+18304 q^{5}+90552 q^{6}+341568 q^{7}+1068928 q^{8}+O\left(q^{9}\right)
\end{aligned}
$$

The factor 4 is due to the automorphism group of each labeled graph of the type 1 .

\section{REFERENCES}

[AEZ16] Jayadev S. Athreya, Alex Eskin, and Anton Zorich. Right-angled billiards and volumes of moduli spaces of quadratic differentials on $\mathbb{C P}^{1}$. Ann. Sci. Éc. Norm. Supér. (4), 49(6):1311-1386, 2016. With an appendix by Jon Chaika.

[BBBM13] J. Böhm, K. Bringmann, A. Buchholz, and H. Markwig. Tropical mirror symmetry for elliptic curves. J. reine angew. Math., Ahead of Print, 2013.

[BCOV94] M. Bershadsky, S. Cecotti, H. Ooguri, and C. Vafa. Kodaira-Spencer theory of gravity and exact results for quantum string amplitudes. Comm. Math. Phys., 165(2):311-427, 1994.

[BO00] S. Bloch and A. Okounkov. The character of the infinite wedge representation. Adv. Math., 149(1):1-60, 2000. 
[CMZ16] D. Chen, M. Möller, and Zagier. Siegel-Veech constants: quasimodularity and large genus limits, 2016, preprint, arXiv:AG/1606.04065.

[Dij95] R. Dijkgraaf. Mirror symmetry and elliptic curves. In The moduli space of curves (Texel Island, 1994), volume 129 of Progr. Math., pages 149-163. Birkhäuser Boston, Boston, MA, 1995.

[EKZ14] A. Eskin, M. Kontsevich, and A. Zorich. Sum of Lyapunov exponents of the Hodge bundle with respect to the Teichmüller geodesic flow. Publ. Math. Inst. Hautes Études Sci., 120:207-333, 2014.

[EM01] A. Eskin and H. Masur. Asymptotic formulas on flat surfaces. Ergodic Theory Dynam. Systems, 21(2):443-478, 2001.

[EMZ03] A. Eskin, H. Masur, and A. Zorich. Moduli spaces of Abelian differentials: the principal boundary, counting problems, and the Siegel-Veech constants. Publ. Math. Inst. Hautes Études Sci., 97:61-179, 2003.

[EO01] A. Eskin and A. Okounkov. Asymptotics of numbers of branched coverings of a torus and volumes of moduli spaces of holomorphic differentials. Invent. Math., 145(1):59103, 2001.

[Gou16] E. Goujard. Volumes of strata of moduli spaces of quadratic differentials: getting explicit values. Ann. Inst. Fourier (Grenoble), 66(6):2203-2251, 2016.

[KO94] S. Kerov and G. Olshanski. Polynomial functions on the set of Young diagrams. $C$. R. Acad. Sci. Paris Sér. I Math., 319(2):121-126, 1994.

[KZ95] M. Kaneko and D. Zagier. A generalized Jacobi theta function and quasimodular forms. In The moduli space of curves (Texel Island, 1994), volume 129 of Progr. Math., pages 165-172. Birkhäuser Boston, Boston, MA, 1995.

[Las08] M. Lassalle. An explicit formula for the characters of the symmetric group. Math. Ann., 340(2):383-405, 2008.

[Li12] S. Li. Feynman graph integrals and almost modular forms. Commun. Number Theory Phys., 6(1):129-157, 2012.

[LZ04] S. K. Lando and A. K. Zvonkin. Graphs on surfaces and their applications, volume 141 of Encyclopaedia of Mathematical Sciences. Springer-Verlag, Berlin, 2004. With an appendix by Don B. Zagier, Low-Dimensional Topology, II.

[Mil03] A. Milas. Formal differential operators, vertex operator algebras and zeta-values. II. J. Pure Appl. Algebra, 183(1-3):191-244, 2003.

[Möl08] M. Möller. Finiteness results for Teichmüller curves. Ann. Inst. Fourier (Grenoble), 58(1):63-83, 2008.

[OO97] A. Okunkov and G. Olshanski. Shifted Schur functions. Algebra i Analiz, 9(2):73-146, 1997.

[OP06] A. Okounkov and R. Pandharipande. Gromov-Witten theory, Hurwitz theory, and completed cycles. Ann. of Math. (2), 163(2):517-560, 2006.

[SSZ12] S. Shadrin, L. Spitz, and D. Zvonkine. On double Hurwitz numbers with completed cycles. J. Lond. Math. Soc. (2), 86(2):407-432, 2012.

[Viv13] F. Viviani. Tropicalizing vs. compactifying the Torelli morphism. In Tropical and nonArchimedean geometry, volume 605 of Contemp. Math., pages 181-210. Amer. Math. Soc., Providence, RI, 2013.

[Zag91] D. Zagier. Periods of modular forms and Jacobi theta functions. Invent. Math., 104(3):449-465, 1991.

[Zag16] D. Zagier. Partitions, quasimodular forms, and the Bloch-Okounkov theorem. Ramanujan J., 41(1-3):345-368, 2016.

[Zor02] A. Zorich. Square tiled surfaces and Teichmüller volumes of the moduli spaces of abelian differentials. In Rigidity in dynamics and geometry (Cambridge, 2000), pages 459-471. Springer, Berlin, 2002.

Laboratoire de Mathématiques d'Orsay, Université Paris-Sud, F-91405 Orsay Cedex, France,

E-mail address: elise.goujard@gmail.com

Institut für Mathematik, Goethe-Universität Frankfurt, Robert-Mayer-Str. 6-8, 60325 Frankfurt am Main, Germany,

E-mail address: moeller@math.uni-frankfurt.de 